\title{
Sparticle Spectrum of Large Volume Compactification
}

\author{
Kiwoon Choi ${ }^{a}$, Hans Peter Nilles ${ }^{b}$, Chang Sub Shin $^{a}$, Michele Trapletti $^{b}$ \\ a Department of Physics, Korea Advanced Institute of Science and Technology \\ Daejeon 305-701, Korea \\ ${ }^{b}$ Bethe Center for Theoretical Physics and Physikalisches Institut, Universität Bonn \\ Nussallee 12, D-53115 Bonn, Germany
}

\begin{abstract}
We examine the large volume compactification of Type IIB string theory or its $F$ theory limit and the associated supersymmetry breakdown and soft terms. It is crucial to incorporate the loop-induced moduli mixing, originating from radiative corrections to the Kähler potential. We show that in the presence of moduli mixing, soft scalar masses generically receive a $D$-term contribution of the order of the gravitino mass $m_{3 / 2}$ when the visible sector cycle is stabilized by the $D$-term potential of an anomalous $U(1)$ gauge symmetry, while the moduli-mediated gaugino masses and $A$-parameters tend to be of the order of $m_{3 / 2} / 8 \pi^{2}$. It is noticed also that a too large moduli mixing can destabilize the large volume solution by making it a saddle point.
\end{abstract}




\section{INTRODUCTION}

Supersymmetry (SUSY) breakdown in string theory often requires the presence of fluxes and nonperturbative effects (like gaugino condensation) [1, 2]. The complete picture, however, requires the fixing of many moduli fields. This might be relatively easy to fulfill in the framework of type IIB theory. Three-form fluxes, gaugino condensation (or $D$-brane instantons) and a specific uplifting mechanism [3] lead to a picture where the gravitino mass and the soft supersymmetry breaking terms can be determined explicitly [4, 5]. The smallness of the gravitino mass (compared to the string and Planck scales) in the low energy effective supergravity theory originates from a small constant term $W_{0}$ in the superpotential*. The mediation of SUSY breakdown is a variant of gravity mediation. In the simplest case the contribution to the soft terms is suppressed by the factor $1 / \ln \left(M_{\text {Planck }} / m_{3 / 2}\right)$ compared to the gravitino mass $m_{3 / 2}$ and radiative corrections such as anomaly mediation become competitive, leading to a scheme called mirage mediation [5, 6]. Then the scale of soft terms is set essentially by $m_{3 / 2}$, suppressed by a factor $1 / \ln \left(M_{\text {Planck }} / m_{3 / 2}\right) \sim 1 / 4 \pi^{2}$, and the gravitino mass should thus be in the multi-TeV range.

Recently, alternative metastable local minima of the scalar potential in type IIB theory have been analyzed. They are characterized by large compactification volume, leading to the socalled large volume scenario (LVS) [7]. An attractive feature of this scenario is the fact that a small value of $m_{3 / 2}$ does not require a small constant $W_{0}$ in the superpotential, but can rather be the consequence of large volume suppression. With $W_{0} \sim \mathcal{O}(1)$, we have the approximate relation $M_{\text {Planck }} / M_{\text {string }} \sim M_{\text {string }} / m_{3 / 2}$. Values of $m_{3 / 2}$ in the TeV range would then require the string scale at an intermediate scale around $10^{11} \mathrm{GeV}$. Therefore it might be difficult to incorporate the idea of grand unification (at a scale around $10^{16} \mathrm{GeV}$ ) in the large volume scenario.

Of course, a full understanding of the situation needs a reliable computation of soft SUSY breaking terms and their relation to the gravitino mass, which is not available yet. In a recent paper [8], it has been suggested that the soft terms might be tiny compared to $m_{3 / 2}$, for instance $m_{\text {soft }} \sim m_{3 / 2}^{3 / 2} / M_{\text {Planck }}^{1 / 2}$ or even as small as $m_{\text {soft }} \sim m_{3 / 2}^{2} / M_{\text {Planck. This would then allow }}$ the gravitino mass as large as $10^{11} \mathrm{GeV}$ and the string scale to be of order of $10^{15} \mathrm{GeV}$, which

\footnotetext{
* In this picture, the uplifting potential is exponentially small as it arises from a SUSY breakdown at the tip of warped throat, and then small $W_{0}$ is required to tune the cosmological constant to a nearly vanishing value.
} 
would allow the incorporation of gauge coupling unification at $M_{G U T} \sim 10^{16} \mathrm{GeV}[8]$ in the LVS scheme. In a more recent paper [9], however, it has been argued that a mechanism of "moduli mixing" could destabilize this hierarchy and bring the soft terms closer to the gravitino mass. This is reminiscent of similar discussions in heterotic string theory (as well as Horava-Witten theory), where higher order corrections to gauge kinetic functions and Kähler potential were shown to destabilize such hierarchies between $m_{\text {soft }}$ and $m_{3 / 2}$ [10, 11]. As was pointed out in [12], it is difficult to generate a nonperturbative superpotential for the visible sector 4-cycle supporting chiral matter fields, which points toward a $D$-term stabilization of the visible sector 4-cycle in LVS [8]. Then there might be additional contribution to soft terms arising from the $D$-term scalar potential.

In this paper we would like to analyze the large volume compactification and the associated soft terms in a class of LVS-theories taking into account the potential "instabilities" of this scenario. Radiative corrections to the Kähler potential and the resulting moduli mixings are shown to become important for the values of soft terms, completely dominating the contributions of order $m_{\text {soft }} \sim m_{3 / 2}^{3 / 2} / M_{\text {Planck }}^{1 / 2}$ discussed in [8]. We also stress the importance of the $D$-terms along the visible sector 4-cycle in the LVS-models. They tend to dominate the soft scalar mass terms and give a contribution of the order of the gravitino mass $m_{3 / 2}$. Gaugino masses and A-parameters do not receive $D$-term contributions, and generically tend to be loopsuppressed, being of the order of $\mathcal{O}\left(m_{3 / 2} / 8 \pi^{2}\right)$. With these contributions from moduli mixings, if the gravitino mass were of the order of $10^{11} \mathrm{GeV}$ as conjectured in [8] (in order to accommodate the unification scale $M_{G U T} \sim 10^{16} \mathrm{GeV}$ with $\left.W_{0} \sim \mathcal{O}(1)\right)$, severe fine-tuning of the Kähler potential at the multi-loop level would be required to keep the soft terms in the TeV range. We would therefore argue that the gravitino mass should not exceed the (multi)-TeV range.

This paper is organized as follows. In section 2, we revisit the LVS-scheme while incorporating the moduli redefinition discussed in [9]. We also include a discussion of the stability of the large volume solution in the presence of moduli mixing. Section 3 discusses the $D$-term stabilization of the visible sector cycle with an explicit scheme to stabilize the remained $D$-flat direction which is parameterized in this case by $U(1)_{A}$-charged (but MSSM singlet) matter fields breaking a global Peccei-Quinn symmetry spontaneously. This scheme naturally generates an intermediate axion scale in LVS, and can be implemented in other scenarii with a high string scale close to the Planck scale. Section 4 is devoted to the discussion of supersymmtry breakdown and resulting soft terms, and conclusions and outlook will be given in section 5 . 


\section{LARGE VOLUME COMPACTIFICATION WITH MODULI MIXING}

In this section, we revisit the large volume scenario (LVS) while incorporating the loopinduced moduli mixing discussed in [9]. To achieve a large compactification volume, one needs at least two Kähler moduli, $T_{1}$ and $T_{2}$, where $T_{1}$ describes a 4-cycle with large volume and $T_{2}$ stands for a small 4-cycle supporting non-perturbative effects such as D3 instantons. An exponentially large vacuum value of $t_{1}=T_{1}+T_{1}^{*}$ is obtained by the competition between the

$\alpha^{\prime}$-correction of $\mathcal{O}\left(1 / t_{1}^{3 / 2}\right)$ and the $D 3$ instanton effect of $\mathcal{O}\left(e^{-a T_{2}}\right)$, yielding $t_{1}^{3 / 2} \sim\left|e^{a T_{2}}\right|$. In $4 \mathrm{D}$ effective SUGRA of LVS, the 4 D Planck scale $M_{\text {Planck }} \equiv 1 / \sqrt{8 \pi G_{N}}$ and the cutoff scale $\Lambda$ of local dynamics on small 4-cycle differ by certain powers of the compactification volume. For instance, in type IIB theory we have

$$
\frac{M_{\text {Planck }}^{2}}{M_{\text {string }}^{2}} \sim \mathcal{V}_{C Y} \sim t_{1}^{3 / 2},
$$

where $\mathcal{V}_{C Y}$ denotes the Calabi-Yau volume in the string length unit with $M_{\text {string }}=1$, and

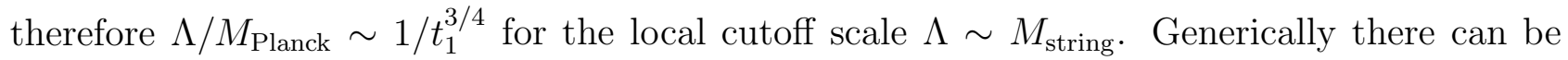
radiative corrections localized on a small 4-cycle, which are controlled by a coupling inversely proportional to the 4-cycle volume $t_{2}=T_{2}+T_{2}^{*}$, and also depend logarithmically on the local cutoff scale $\Lambda$. Since $M_{\text {Planck }}$ is the natural mass scale of $4 \mathrm{D}$ effective SUGRA, including those quantum corrections in the Kähler potential and/or the gauge kinetic functions would require a redefinition of $t_{2}$, involving $\ln \left(M_{\text {Planck }} / \Lambda\right) \propto \ln t_{1}[9]$ as

$$
t_{2} \rightarrow \tilde{t}_{2}=t_{2}-\alpha_{2} \ln t_{1}
$$

where $\alpha_{2}$ is a parameter representing the size of the quantum corrections that lead to the above moduli-mixing. (Note that this is not a redefinition of the chiral superfield, but a redefinition of the scalar component of the chiral superfield.) In fact, a similar phenomenon has been noticed in heterotic string/ $M$ theory context, i.e. a redefinition of the heterotic string dilaton [13]

$$
s \rightarrow s-\alpha \ln t
$$

which is required to accommodate the loop threshold correction to 4D gauge coupling [14] where $s=S+S^{*}$ is the heterotic string dilaton and $t=T+T^{*}$ is a Kähler modulus in underlying heterotic string compactification. In the heterotic $M$-theory limit, $s$ corresponds to the small volume of $6 \mathrm{D}$ internal space, while $t$ describes the large length of the 11-th dimension. Then 
the heterotic redefinition (3) in the limit $t \gg s$ can have a similar geometric interpretation as the type IIB field redefinition (2) in LVS.

To proceed, following [7, 8], we assume that all complex structure moduli and the string dilaton are stabilized by fluxes at a supersymmetric solution, and those flux-stabilized moduli can be integrated out without affecting the subsequent stabilization of Kähler moduli. ${ }^{\dagger}$ In the following, unless specified, we set the $4 \mathrm{D}$ Planck scale (in the Einstein frame) $M_{\text {Planck }}=1$.

Since we are interested in the large volume limit $t_{1} \gg 1$, it is convenient to expand the Kähler potential of the model in (appropriate) powers of $1 / t_{1}$. Then, after the dilaton and complex structure moduli are integrated out, the Kähler potential and superpotential of $T_{i}$ $(i=1,2)$ are given by $[9]$

$$
\begin{aligned}
K & =-3 \ln t_{1}+\frac{2\left(\tilde{t}_{2}^{3 / 2}-\xi_{\alpha^{\prime}}\right)}{t_{1}^{3 / 2}}+\mathcal{O}\left(1 / t_{1}^{3}\right), \\
W & =W_{0}+A e^{-a T_{2}}
\end{aligned}
$$

where

$$
\tilde{t}_{2}=t_{2}-\alpha_{2} \ln t_{1} \quad\left(t_{i}=T_{i}+T_{i}^{*}\right) .
$$

The parameter $\xi_{\alpha^{\prime}}$ in the Kähler potential represents the $\alpha^{\prime}$-correction, and $\alpha_{2}$ parameterizes the radiative corrections that lead to the loop-induced redefinition of $t_{2}$. Here we assume that $A e^{-a T_{2}}$ is induced by $D 3$ instantons, so

$$
\frac{a t_{2}}{2}=\text { Euclidean action of } D 3 \text { instanton. }
$$

The constant $W_{0}$ in the superpotential might arise from 3-form fluxes, and is assumed to be of order unity in LVS. As we will see, for the model of (4), we have

$$
\frac{m_{3 / 2}}{M_{\text {Planck }}}=e^{K / 2}|W| \sim \frac{\left|W_{0}\right|}{t_{1}^{3 / 2}} \sim\left|A e^{-a T_{2}}\right|
$$

regardless of the value of $W_{0}$. As a result, the value of $D 3$ instanton action is given by

$$
\frac{a t_{2}}{2} \sim \ln \left(M_{\text {Planck }} / m_{3 / 2}\right) \sim 4 \pi^{2}
$$

\footnotetext{
$\dagger^{\dagger}$ Once $T_{i}$ are stabilized at the SUSY-breaking vacuum, nonzero $F$-components of the dilaton and complex structure moduli do appear as well. However their effects are subleading compared to those of $F^{T_{i}}$ in the large volume limit $t_{1} \gg 1$.
} 
regardless of whether $W_{0} \sim \mathcal{O}(1)$ or hierarchically small. On the other hand, with Eq. (5), $\alpha_{2} / t_{2}$ corresponds to a loop suppression factor on small 4-cycle, and thus is expected to be $\mathcal{O}\left(1 / 8 \pi^{2}\right)$, which implies

$$
a \alpha_{2}=\mathcal{O}(1)
$$

With appropriate $R$-transformation and axionic shift $\operatorname{Im}\left(T_{2}\right) \rightarrow \operatorname{Im}\left(T_{2}\right)+$ constant, we can always make $W_{0}$ and $A$ to be real positive parameters. In the following, we will take such a field basis in which $W_{0}$ and $A$ are real and positive.

Let us now examine the stabilization of Kähler moduli in the 4D effective SUGRA model of (44). The scalar potential of the model is given by

$$
V_{\text {SUGRA }}=e^{K}\left\{K^{I \bar{J}} D_{I} W\left(D_{J} W\right)^{*}-3|W|^{2}\right\}
$$

Assuming $t_{1} \gg 1$ and also $a \tilde{t}_{2}=\mathcal{O}\left(8 \pi^{2}\right)$ as indicated by (8), we can solve the equations of motion

$$
\partial_{t_{1}} V_{\mathrm{SUGRA}}=\partial_{t_{2}} V_{\mathrm{SUGRA}}=0
$$

to find

$$
\begin{aligned}
\frac{t_{1}^{3 / 2}}{W_{0}} & =\frac{e^{a t_{2} / 2}}{a A} \xi_{\alpha^{\prime}}^{1 / 3}\left(\frac{3}{2}-\frac{21+8 a \alpha_{2}}{12 a \tilde{t}_{2}}+\mathcal{O}\left(\frac{1}{\left(a \tilde{t}_{2}\right)^{2}}\right)\right), \\
\tilde{t}_{2}^{3 / 2} & =\xi_{\alpha^{\prime}}\left(1+\frac{3-13 a \alpha_{2}}{3 a \tilde{t}_{2}}+\mathcal{O}\left(\frac{1}{\left(a \tilde{t}_{2}\right)^{2}}\right)\right),
\end{aligned}
$$

where the solution is expanded in powers of $1 / a \tilde{t}_{2}$. The above solution shows that $t_{1}$ can indeed have an exponentially large value for the parameter values given that $a t_{2} \gg 1$. Using the above solution, we can find the following moduli mass spectrum:

$$
\begin{aligned}
& m_{t_{1}}^{2} \approx\left(3-a \alpha_{2}\right) \frac{\xi_{\alpha^{\prime}}}{a \tilde{t}_{2}} \frac{m_{3 / 2}^{2}}{t_{1}^{3 / 2}}, \quad m_{a_{1}}^{2}=0, \\
& m_{t_{2}}^{2} \approx m_{a_{2}}^{2} \approx\left(a \tilde{t}_{2}\right)^{2} m_{3 / 2}^{2}
\end{aligned}
$$

implying that we need

$$
a \alpha_{2}<3
$$

in order for the solution (12) to be a (meta) stable local minimum. 
On the other hand, the form of the Kähler potential and superpotential in (4) is valid only when both $t_{2}$ and $\tilde{t}_{2}$ are positive in the convention that $a$ is positive, which corresponds to the condition that both the D3 instanton action and the moduli Kähler metric are positive. Combined with the vacuum solution (12), this leads to

$$
\frac{3 \tilde{t}_{2}}{t_{2}}=3-a \alpha_{2}+\frac{2 \alpha_{2}}{t_{2}}\left(\ln \left(1 / w_{0}\right)+\mathcal{O}(1)\right)>0 .
$$

For the case with $W_{0} \sim \mathcal{O}(1)$, since $\alpha_{2} / t_{2}=\mathcal{O}\left(1 / 8 \pi^{2}\right)$, the vacuum stability condition (14) is satisfied for most part of the parameter region satisfying the above condition. Although $W_{0}$ is assumed to be $\mathcal{O}(1)$ in usual LVS, it is often required to be hierarchically small in order to accommodate both the gauge unification at $10^{16} \mathrm{GeV}$ and the soft SUSY breaking masses $\sim 1 \mathrm{TeV}$. For instance, in the presence of moduli mixing, our discussion in the next section implies that sfermions in the visible sector can get a soft mass of $\mathcal{O}\left(m_{3 / 2}\right)$ from the $D$-term contribution, while the gaugino masses are of $\mathcal{O}\left(m_{3 / 2} / 8 \pi^{2}\right)$. In this case, demanding gauge unification at $10^{16} \mathrm{GeV}$ and the weak scale size of gaugino masses, one finds $M_{\text {string }} \sim 10^{15}$ $\mathrm{GeV}$ and $m_{3 / 2} \sim 10 \mathrm{TeV}$, which correspond to $t_{1} \sim 10^{3}$ and $W_{0} \sim 10^{-10}$ (see (11) and (7)). In such case of small $W_{0}$, there can be a sizable part of parameter space which satisfies (15), but is excluded by the stability condition (14).

For $a \alpha_{2}<3$, the large volume solution (12) corresponds to an AdS vacuum (at least at tree level) as it gives the vacuum energy density

$$
V_{\text {vacuum }} \approx-\left(3-a \alpha_{2}\right) \frac{\xi_{\alpha^{\prime}}}{a \tilde{t}_{2}} \frac{m_{3 / 2}^{2}}{t_{1}^{3 / 2}}
$$

Then, to achieve a phenomenologically viable de-Sitter vacuum, one might need an uplifting potential induced by additional sources of SUSY breaking ${ }^{\ddagger}$. Since $m_{t_{2}}=\mathcal{O}\left(8 \pi^{2} m_{3 / 2}\right)$ as indicated by (13), such an uplifting potential would not significantly affect the vacuum solution along the $t_{2}$-direction, however it can cause a large shift of $t_{1}$, and even might destabilize the solution.

It is unclear yet if a successful uplifting sector can be introduced within the framework of large volume compactification of type IIB theory or its $F$-theory limit ${ }^{\S}$. Here we take a

\footnotetext{
$\ddagger$ Alternatively, one can assume that (quadratically divergent) radiative corrections to the vacuum energy density do the job of uplifting [15].

$\S$ In KKLT compactification with warped throat, a successful uplifting can be achieved by anti- $D 3$ branes (or any SUSY breaking branes) stabilized at the tip of throat.
} 
phenomenological approach, simply introducing an uplifting potential inversely proportional to certain powers of the CY volume $\mathcal{V}_{C Y}$ :

$$
\Delta V_{\text {lift }}=\frac{D_{0}}{\mathcal{V}_{C Y}^{n_{0}}}=\frac{D_{0}}{t_{1}^{3 n_{0} / 2}}\left(1+\mathcal{O}\left(\frac{1}{t_{1}^{3 / 2}}\right)\right),
$$

where $n_{0}$ is a positive rational number and $D_{0}$ is a positive constant which should be tuned to make the final vacuum energy density nearly zero. We then find that the stationary solution of the total potential $V_{\mathrm{TOT}}=V_{\mathrm{SUGRA}}+\Delta V_{\text {lift }}$ is given by

$$
\begin{aligned}
\frac{t_{1}^{3 / 2}}{W_{0}} & =\frac{e^{a t_{2} / 2}}{a A} \xi_{\alpha^{\prime}}^{1 / 3}\left(\frac{3}{2}-\frac{\left(21-9 n_{0}\right)+\left(8-2 n_{0}\right) a \alpha_{2}}{4\left(3-n_{0}\right) a \tilde{t}_{2}}+\mathcal{O}\left(\left(a \tilde{t}_{2}\right)^{-2}\right)\right) \\
\tilde{t}_{2}^{3 / 2} & =\xi_{\alpha^{\prime}}\left(1+\frac{3-\left(13-4 n_{0}\right) a \alpha_{2}}{\left(3-n_{0}\right) a \tilde{t}_{2}}+\mathcal{O}\left(\left(a \tilde{t}_{2}\right)^{-2}\right)\right),
\end{aligned}
$$

showing that the qualitative feature of moduli vacuum values is untouched by the uplifting potential. Considering the moduli masses, we find $m_{t_{1}}$ is modified

$$
m_{t_{1}}^{2}=\left(3-n_{0}\right)\left(3-a \alpha_{2}\right) \frac{\xi_{\alpha^{\prime}}}{t_{1}^{3 / 2}} \frac{m_{3 / 2}^{2}}{a \tilde{t}_{2}}
$$

while the other moduli masses are the same as those in (13). Since the uplifting is required only for $a \alpha_{2}<3$ (see the vacuum energy density (16) ), this form of the large volume modulus mass implies that the stationary solution (18) becomes a saddle point when $n_{0}>3$, for which the uplifting potential is too steep to give a local de-Sitter minimum.

\section{D-TERM STABILIZATION OF THE MSSM CYCLE MODULUS}

It has been pointed out in [12] that a 4-cycle supporting chiral matter fields can not have a D3 instanton superpotential. This implies that the D3-instanton 4-cycle described by the Kähler modulus $T_{2}$ can not be identified as the visible sector 4-cycle supporting the MSSM gauge and matter fields. The large volume 4-cycle also can not be identified as the visible sector cycle as it would give a too small SM gauge coupling $g_{S M}^{2} \sim 1 / t_{1}$. With this observation, a third 4-cycle has been introduced in [8] to accommodate the MSSM sector under the assumption that the corresponding Kähler modulus $T_{3}$ is stabilized by a $D$-term potential.

As there is no instanton superpotential of the form $e^{-b T_{3}}$, the $D$-term stabilization of $T_{3}$ is indeed a natural direction to be explored. For this, we need a $D$-term which depends on $t_{3}=$ $T_{3}+T_{3}^{*}$ even in the limit that other (gauge charged) matter fields are all vanishing. This would 
be achieved by having an anomalous $U(1)$ symmetry under which $T_{3}$ transforms nonlinearly [16], so that the associated anomaly is canceled by the Green-Schwarz (GS) mechanism [17]:

$$
U(1)_{A}: \quad V_{A} \rightarrow V_{A}+\Lambda_{A}+\Lambda_{A}^{*}, \quad T_{3} \rightarrow T_{3}+\delta_{G S} \Lambda_{A}, \quad \Phi_{i} \rightarrow e^{-2 q_{i} \Lambda_{A}} \Phi_{i},
$$

where $V_{A}$ is the vector superfield containing the $U(1)_{A}$ gauge field, $\Lambda_{A}$ is a chiral superfield parameterizing the $U(1)_{A}$ transformation on $N=1$ superspace, $\delta_{G S}$ is a constant of $\mathcal{O}\left(1 / 8 \pi^{2}\right)$ (under a proper normalization of $T_{3}$ ), and finally $\Phi_{i}$ stand for generic chiral matter superfields with $U(1)_{A}$ charge $q_{i}$. The gauge boson mass and the $D$-term of $U(1)_{A}$ are given by

$$
\begin{aligned}
M_{A}^{2} & =2 g_{A}^{2} \eta^{I} \eta^{\bar{J}} K_{I \bar{J}} \equiv 2 g_{A}^{2}\left(M_{G S}^{2}+M_{P Q}^{2}\right), \\
D_{A} & =-\eta^{I} K_{I} \equiv \xi_{F I}+\tilde{M}_{P Q}^{2},
\end{aligned}
$$

where $g_{A}$ is the $U(1)_{A}$ gauge coupling, $2 \eta^{I}=-\delta \Phi^{I} / \delta \Lambda_{A}=\left\{-\delta_{G S}, 2 q_{i} \Phi_{i}\right\}$ denote the holomorphic Killing vector field generating an infinitesimal $U(1)_{A}$ transformation of chiral superfields $\Phi^{I}=\left\{T_{3}, \Phi_{i}\right\}$, and

$$
\begin{aligned}
M_{G S}^{2} & \left.\equiv\left(\frac{\delta_{G S}}{2}\right)^{2}\left\langle\frac{\partial^{2} K}{\partial T_{3} \partial T_{3}^{*}}\right\rangle\right|_{\Phi_{i}=0}, \\
\xi_{F I} & \left.\equiv \frac{\delta_{G S}}{2}\left\langle\frac{\partial K}{\partial T_{3}}\right\rangle\right|_{\Phi_{i}=0} .
\end{aligned}
$$

Note that $M_{G S}^{2}$ corresponds to the $U(1)_{A}$ gauge boson mass-square in the limit $\Phi_{i}=0$, which originates from the Stückelberg mechanism associated with the $U(1)_{A}$ transformation of $\operatorname{Im}\left(T_{3}\right)$, while $M_{P Q}^{2} \propto\left\langle\Phi_{i}^{*} \Phi_{i}\right\rangle$ stands for the contribution to $M_{A}^{2}$ from the nonzero vacuum values of the $U(1)_{A}$ charged matter fields $\Phi_{i}$. (Here we use the subscript "PQ" as $M_{P Q}$ corresponds to the scale of spontaneous breaking of a $U(1)$ Peccei-Quinn symmetry [18].) The nonlinear transformation of $T_{3}$ under $U(1)_{A}$ gives rise to the moduli-dependent Fayet-Illiopoulos (FI) term $\xi_{F I}$ which should be canceled by the $D$-term contribution $\tilde{M}_{P Q}^{2}$ from the vacuum values of $\Phi_{i}$.

The explicit realization of the $D$-term stabilization of $T_{3}$ depends on the relative size of $\xi_{F I}$ compared to $M_{G S}^{2}[19,20]$, which would identify the $D$-flat direction. If $T_{3}$ is stabilized at a point with $\left|\xi_{F I}\right| \gg M_{G S}^{2}$, the $U(1)_{A}$ gauge boson gets most of its mass from the vacuum values of $\Phi_{i}$ since $\tilde{M}_{P Q}^{2} \approx-\xi_{F I}$ along the $D$-flat direction and also generically $\left|M_{P Q}^{2}\right| \sim\left|\tilde{M}_{P Q}^{2}\right|$. In this case, the $D$-term potential fixes essentially a combination of $U(1)_{A}$-charged matter fields $\Phi_{i}$, while leaving $T_{3}$ as an unfixed $D$-flat direction. This would be the case when $T_{3}$ is stabilized at 
a geometric regime where $t_{3} \sim 1 / g_{G U T}^{2}=\mathcal{O}(1)$ and the Kähler potential obeys a simple scaling behavior $\partial K / \partial t_{3} \sim t_{3} \partial^{2} K / \partial^{2} t_{3}$, giving

$$
\frac{\xi_{F I}}{M_{G S}^{2}}=\left.\frac{2}{\delta_{G S}}\left\langle\frac{\partial_{T_{3}} K}{\partial_{T_{3}} \partial_{T_{3}^{*}} K}\right\rangle\right|_{\Phi_{i}=0} \sim \frac{t_{3}}{\delta_{G S}} \sim \mathcal{O}\left(8 \pi^{2}\right) .
$$

Alternatively, $T_{3}$ might be stabilized at or near a (singular) point with vanishing FI term [8, 9]. In such case, it is possible to have

$$
\xi_{F I} \sim \tilde{M}_{P Q}^{2} \sim M_{P Q}^{2} \ll M_{G S}^{2}
$$

so that the $U(1)_{A}$ gauge boson mass-square is dominated by the Stückelberg contribution $M_{G S}^{2}$. Then $t_{3}$ is stabilized as desired by the $D$-term potential of $U(1)_{A}$, while the unfixed $D$-flat direction is described mostly by a combination of $\Phi_{i}$. This limit is particularly interesting since it offers the possibility that $M_{P Q}$ is far below the string scale. As we will see, in this case, $M_{P Q}$ can be identified as the scale of spontaneous breakdown of an anomalous global symmetry which can solve the strong CP problem [18, 21, 22]. One can then have a QCD axion with phenomenologically favored decay constant $M_{P Q} \sim 10^{9}-10^{12} \mathrm{GeV}$ [23] even when the string scale is close to $M_{\text {Planck }}$. Another interesting feature of this case is that the $D$ flat direction can be easily stabilized by the combined effects of supersymmetry breaking and appropriate tree-level superpotential of $\Phi_{i}$. Thus, in the following, we will focus on the case with $\xi_{F I} \ll M_{G S}^{2}$.

To proceed, let $V_{a}$ denote the MSSM gauge superfields localized on $D$-branes wrapping the visible sector 4-cycle. Including $V_{A}$ and the chiral matter fields $\Phi_{i}$ together, and keeping only the leading order terms in the expansion in $1 / t_{1}$, the Kähler potential, superpotential and gauge kinetic functions of the model are given by

$$
\begin{aligned}
K & =K_{0}\left(t_{1}, t_{2}, t_{3}\right)+Z_{i} \Phi_{i}^{*} e^{2 q_{i} V_{A}} \Phi_{i}+\mathcal{O}\left(|\Phi|^{4}\right), \\
W & =\tilde{W}_{0}\left(T_{I}\right)+\frac{1}{3 !} \lambda_{i j k} \Phi_{i} \Phi_{j} \Phi_{k}+\mathcal{O}\left(\Phi^{4}\right), \\
f_{a} & =\gamma_{a}+k_{a} T_{3}, \quad f_{A}=\gamma_{A}+k_{A} T_{3},
\end{aligned}
$$

where

$$
\begin{aligned}
& K_{0}=-3 \ln t_{1}+\frac{2\left(\tilde{t}_{2}^{3 / 2}-\xi_{\alpha^{\prime}}\right)}{t_{1}^{3 / 2}}+\frac{1}{2 t_{1}^{p}}\left(\tilde{t}_{3}^{2}+\mathcal{O}\left(\tilde{t}_{3}^{3}\right)\right) \\
& \tilde{W}_{0}=W_{0}+A e^{-a T_{2}}
\end{aligned}
$$


for

$$
\begin{aligned}
& t_{i}=T_{i}+T_{i}^{*} \quad(i=1,2,3), \\
& \tilde{t}_{2}=t_{2}-\alpha_{2} \ln t_{1}, \quad \tilde{t}_{3}=t_{3}-\alpha_{3} \ln t_{1}-\delta_{G S} V_{A},
\end{aligned}
$$

and $f_{a}$ and $f_{A}$ denote the holomorphic gauge kinetic functions for the MSSM and $U(1)_{A}$ gauge superfields, respectively. The constants $\gamma_{a}$ and $\gamma_{A}$ in the gauge kinetic functions might be induced by the vacuum value of the type IIB string dilaton, and they have a value of order unity (or smaller) in general. As was noticed in [9, 13], generically it is expected that the radiative corrections on the visible sector 4 -cycle require a redefinition of $t_{3}$ depending on $\ln \left(M_{\text {Planck }} / \Lambda\right) \propto \ln t_{1}$, where $\Lambda$ is the local cutoff scale :

$$
t_{3} \rightarrow t_{3}-\alpha_{3} \ln t_{1}
$$

The form of the Kähler potential of $t_{3}$ is dictated by the condition that $t_{3}$ is stabilized near the point with vanishing FI-term, which can be defined as

$$
\left.\frac{\partial K}{\partial \tilde{t}_{3}}\right|_{\tilde{t}_{3}=0}=0
$$

for the $U(1)_{A}$-invariant combination $\tilde{t}_{3}$ including the effect of moduli mixing. For simplicity, here we assume that the Kähler metric of $t_{3}$ is independent of the second Kähler modulus $t_{2}$, however all of our subsequent discussions are valid even when the Kähler metric of $t_{3}$ is given by a generic function of $t_{2}$. As for the power $p$ of $t_{1}$ in the Kähler metric of $t_{3}$, one can consider two possibilities:

$$
p=3 / 2 \text { or } 1
$$

If the local cutoff scale of $T_{3}$ is given by the string scale, $p=3 / 2$ would be the correct choice [9]. On the other hand, if the Kähler metric of $T_{3}$ behaves like those of $\Phi_{i}$, one would have $p=1$ for which the local cutoff scale corresponds to the winding mode scale $M_{*} \sim t_{1}^{1 / 4} M_{\text {string }}$. Note that $M_{G S}$ takes a different value depending upon the value of $p$ :

$$
M_{G S} \sim \delta_{G S} M_{\text {string }}(p=3 / 2) \quad \text { or } \quad \delta_{G S} M_{*}(p=1) .
$$

Yukawa couplings of the canonically normalized matter fields localized on the MSSM 4-cycle should not have any power-law dependence on the bulk CY volume, and thus no power-law dependence on $t_{1}$. Under this requirement, the matter Kähler metric is given by

$$
Z_{i}=\frac{1}{t_{1}} \mathcal{Y}_{i}\left(\hat{t}_{2}, \hat{t}_{3}\right)
$$


where

$$
\hat{t}_{2}=t_{2}-\beta_{2} \ln t_{1}, \quad \hat{t}_{3}=t_{3}-\beta_{3} \ln t_{1}-\delta_{G S} V_{A}
$$

for the moduli mixing parameters $\beta_{2,3}$ which generically can differ from $\alpha_{2,3}$ that describe the moduli mixing in $K_{0}$. We can always choose the normalization convention of $\Phi_{i}$ to make $\mathcal{Y}_{i}$ have a vacuum value of $\mathcal{O}(1)$, and also take the normalization convention of $T_{1}$ and $T_{3}$ for which

$$
\xi_{\alpha^{\prime}}=\mathcal{O}(1), \quad k_{a, A}=\mathcal{O}(1) .
$$

Then the large volume solution (12) (or (18) in the presence of uplifting potential) and the instanton action (8) indicate that the vacuum value of $t_{2}$ is of $\mathcal{O}(1)$ and the parameter $a=$ $\mathcal{O}\left(\ln \left(M_{\text {Planck }} / m_{3 / 2}\right)\right)$ in our convention. Since the $U(1)_{A}$-variation of the gauge kinetic function should be canceled by the loops of $U(1)_{A}$-charged fermions, under the assumption that $U(1)_{A}$ charges of chiral matter fields are generically of $\mathcal{O}(1)$, we find $\delta_{G S}=\mathcal{O}\left(1 / 8 \pi^{2}\right)$. The parameters describing the loop-induced moduli redefinition, i.e. $\alpha_{2,3}$ and $\beta_{2,3}$, are expected to have a similar size of $\mathcal{O}\left(1 / 8 \pi^{2}\right)$. Summarizing the size of model parameters in our convention, we have

$$
\begin{aligned}
\delta_{G S} & \sim \alpha_{2,3} \sim \beta_{2,3}=\mathcal{O}\left(\frac{1}{8 \pi^{2}}\right), \\
a & =\mathcal{O}\left(\ln \left(M_{\text {Planck }} / m_{3 / 2}\right)\right)=\mathcal{O}\left(8 \pi^{2}\right) .
\end{aligned}
$$

With the Kähler potential given in (25), the $U(1)_{A}$ gauge boson mass and the $D$-term are given by

$$
\begin{aligned}
M_{A}^{2} & =2 g_{A}^{2}\left(M_{G S}^{2}+M_{P Q}^{2}\right), \\
D_{A} & =\xi_{F I}+\tilde{M}_{P Q}^{2}
\end{aligned}
$$

where

$$
\begin{aligned}
& M_{G S}^{2}=\left(\frac{\delta_{G S}}{2}\right)^{2} \frac{1}{t_{1}^{p}}, \quad \xi_{F I}=\frac{\delta_{G S}}{2} \frac{\tilde{t}_{3}}{t_{1}^{p}} \\
& M_{P Q}^{2}=\sum_{i}\left(q_{i}^{2} Z_{i}-q_{i} \delta_{G S} \partial_{t_{3}} Z_{i}+\left(\frac{\delta_{G S}}{2}\right)^{2} \partial_{t_{3}}^{2} Z_{i}\right)\left\langle\Phi_{i}^{*} e^{2 q_{i} V_{A}} \Phi_{i}\right\rangle \\
& \tilde{M}_{P Q}^{2}=-\sum_{i}\left(q_{i} Z_{i}-\frac{\delta_{G S}}{2} \partial_{t_{3}} Z_{i}\right)\left\langle\Phi_{i}^{*} e^{2 q_{i} V_{A}} \Phi_{i}\right\rangle .
\end{aligned}
$$

Here we use units with $M_{\text {Planck }}=1$. The vacuum values of the gauge-invariant combinations $\Phi_{i}^{*} e^{2 q_{i} V_{A}} \Phi_{i}$ will be determined later by the combined effects of supersymmetry breaking and the $F$-term scalar potential. 
Since there is no D3 instanton effect of the form $e^{-b T_{3}}$ [12], the Kähler potential and the superpotential are invariant under the axionic shift of $T_{3}$ :

$$
U(1)_{T_{3}}: \quad T_{3} \rightarrow T_{3}+\text { imaginary constant, }
$$

which is explicitly broken by the variation of the holomorphic gauge kinetic functions. Due to the anomalous $U(1)_{A}$ gauge symmetry, this axionic shift symmetry is equivalent to the global $U(1)_{P Q}$ symmetry under which

$$
U(1)_{P Q}: \quad \Phi \rightarrow e^{i q_{i} \alpha} \Phi_{i}
$$

where $q_{i}$ is the $U(1)_{A}$ charge of $\Phi_{i}$, and $\alpha$ is a real constant. The Green-Schwarz anomaly cancelation [17] for $U(1)_{A}$ requires

$$
\frac{1}{2 \pi^{2}} \sum_{i} q_{i} \operatorname{Tr}\left(T_{a}^{2}\left(\Phi_{i}\right)\right)=k_{a} \delta_{G S}, \quad \frac{1}{2 \pi^{2}} \sum_{i} q_{i}^{3}=k_{A} \delta_{G S}
$$

where $k_{a}$ and $k_{A}$ are the coefficients of $T_{3}$ in the gauge kinetic functions $f_{a}$ and $f_{A}$ in (25). This means that $U(1)_{P Q}$ is an anomalous global symmetry which can solve the strong CP problem by the axion mechanism [18, 21, 22]. Obviously $M_{P Q} \sim \tilde{M}_{P Q}$ corresponds to the scale where $U(1)_{P Q}$ is spontaneously broken, and thus to the axion decay constant which is constrained to be above $10^{9} \mathrm{GeV}$ by astrophysical considerations [23]:

$$
M_{P Q} \geq 10^{9} \mathrm{GeV}
$$

If $t_{3}$ is stabilized by the $D$-term potential, the Kähler modulus superfield $T_{3}$ becomes a part of the massive $U(1)_{A}$ vector superfield. As a result, its equation of motion is encoded in that of $V_{A}$, which takes the form

$$
\frac{\partial K}{\partial V_{A}}=\mathcal{O}\left(\mathcal{D}^{2} \overline{\mathcal{D}}^{2} V_{A}\right)
$$

where $\mathcal{D}^{2}=\mathcal{D}^{\alpha} \mathcal{D}_{\alpha}$ for the superspace covariant derivative $\mathcal{D}_{\alpha}$. Here the right-hand-side of the above equation of motion comes from the variation of the gauge kinetic term in the $N=1$ superspace. As long as $m_{3 / 2} \ll M_{G S}$, this part can be safely ignored in the discussion of moduli stabilization and SUSY breakdown. For the Kähler potential (25), the superfield equation (42) is given by

$$
4 M_{G S}^{2}\left(V_{A}-\frac{t_{3}}{\delta_{G S}}+\frac{\alpha_{3}}{\delta_{G S}} \ln t_{1}\right)+\Phi_{i}^{*} e^{2 q_{i} V_{A}} \Phi_{i}\left(2 q_{i} Z_{i}-\delta_{G S} \frac{\partial Z_{i}}{\partial \hat{t}_{3}}\right)=\mathcal{O}\left(\mathcal{D}^{2} \overline{\mathcal{D}}^{2} V_{A}\right)
$$


The Kähler potential in (26) assumes

$$
\tilde{t}_{3}=t_{3}-\alpha_{3} \ln t_{1}-\delta_{G S} V_{A} \ll 1
$$

The superfield equation (43) suggests that this condition is fulfilled if the matter fields are stabilized as

$$
\left\langle Z_{i} \Phi_{i}^{*} e^{2 q_{i} V_{A}} \Phi_{i}\right\rangle \sim M_{P Q}^{2} \ll \frac{M_{G S}^{2}}{\delta_{G S}}
$$

For simplicity, here we take a stronger condition:

$$
M_{P Q}^{2} \ll M_{G S}^{2}
$$

since the analysis for such case is rather straightforward. In the appendix, we will show that similar results are obtained even when $M_{P Q}^{2} \gtrsim M_{G S}^{2}$ as long as $M_{P Q}^{2} \ll M_{G S}^{2} / \delta_{G S}$.

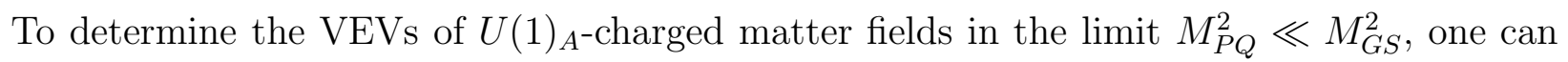
integrate out the massive $U(1)_{A}$ vector superfield including $T_{3}$, and examine the stabilization of $\Phi_{i}$ based on the resulting low energy effective theory [19, 20]. For this, it is convenient to make the field redefinition:

$$
V_{A} \rightarrow V_{A}+\frac{T_{3}+T_{3}^{*}}{\delta_{G S}}, \quad \Phi_{i} \rightarrow e^{-2 q_{i} T_{3} / \delta_{G S}} \Phi_{i},
$$

under which the holomorphic gauge kinetic function is transformed as

$$
f_{a}=\gamma_{a}+k_{a} T_{3} \rightarrow f_{a}^{\mathrm{eff}}=\gamma_{a}+k_{a} T_{3}-\frac{1}{4 \pi^{2}} \sum_{i} \operatorname{Tr}\left(T_{a}^{2}\left(\Phi_{i}\right)\right) \frac{2 q_{i} T_{3}}{\delta_{G S}}=\gamma_{a}
$$

where we have used the anomaly matching condition (40) for the last equality. With this field redefinition, $T_{3}$ is gauged away in (43), and the solution for the $U(1)_{A}$ vector superfield is given by

$$
V_{A}=-\frac{\alpha_{3}}{\delta_{G S}} \ln t_{1}-\left(2 q_{i} Z_{i}^{\mathrm{eff}}-\delta_{G S} \frac{\partial Z_{i}^{\mathrm{eff}}}{\partial \hat{t_{3}}}\right) \frac{\Phi_{i}^{*} \Phi_{i}}{4 M_{G S}^{2}}+\mathcal{O}\left(\left(\frac{Z_{i}^{\mathrm{eff}} \Phi_{i}^{*} \Phi_{i}}{M_{G S}^{2}}\right)^{2}\right)
$$

where

$$
\begin{aligned}
Z_{i}^{\mathrm{eff}} & =\frac{Z_{i}\left(t_{1}, \hat{t}_{2}=t_{2}-\beta_{2} \ln t_{1}, \hat{t}_{3}=\left(\alpha_{3}-\beta_{3}\right) \ln t_{1}\right)}{t_{1}^{2 q_{i} \alpha_{3} / \delta_{G S}}} \\
& =\frac{\mathcal{Y}_{i}\left(\hat{t}_{2}=t_{2}-\beta_{2} \ln t_{1}, \hat{t}_{3}=\left(\alpha_{3}-\beta_{3}\right) \ln t_{1}\right)}{t_{1}^{1+2 q_{i} \alpha_{3} / \delta_{G S}}} .
\end{aligned}
$$


The effective Kähler potential of light fields can be obtained by inserting the above solution into the Kähler potential (25), which gives

$$
\begin{aligned}
K_{\mathrm{eff}} & =K_{0}^{\mathrm{eff}}\left(t_{1}, t_{2}\right)+Z_{i}^{\mathrm{eff}} \Phi_{i}^{*} \Phi_{i}\left(1+\mathcal{O}\left(\frac{Z_{i}^{\mathrm{eff}} \Phi_{i}^{*} \Phi_{i}}{M_{G S}^{2}}\right)\right) \\
& =-3 \ln t_{1}+\frac{2\left(\tilde{t}_{2}^{3 / 2}-\xi_{\alpha^{\prime}}\right)}{t_{1}^{3 / 2}}+Z_{i}^{\mathrm{eff}} \Phi_{i}^{*} \Phi_{i}\left(1+\mathcal{O}\left(\frac{Z_{i}^{\mathrm{eff}} \Phi_{i}^{*} \Phi_{i}}{M_{G S}^{2}}\right)\right) .
\end{aligned}
$$

Note that

$$
M_{P Q}^{2} \sim\left\langle Z_{i} \Phi_{i}^{*} e^{2 q_{i} V_{A}} \Phi_{i}\right\rangle \sim\left\langle Z_{i}^{\mathrm{eff}} \Phi_{i}^{*} \Phi_{i}\right\rangle
$$

and therefore the above effective Kähler potential provides a controllable description of low energy physics in the limit $M_{P Q}^{2} \ll M_{G S}^{2}$.

Let us now present a simple model which fixes the axion scale $M_{P Q}$ by the combined effects of SUSY breaking and a Planck-scale-suppressed term in the superpotential. This type of model provides a setup giving the axion scale in the phenomenologically desirable range $10^{9}$ $\mathrm{GeV} \leq M_{P Q} \leq 10^{12} \mathrm{GeV}$ even when the string scale is close to the Planck scale. To have $M_{P Q} \gtrsim 10^{9} \mathrm{GeV}, U(1)_{P Q}$ should be broken dominantly by $U(1)_{A}$-charged but MSSM-singlet matter fields. As a kind of minimal example, we introduce two such matter fields, $X$ and $Y$, with the following Kähler potential and superpotential [24]:

$$
\begin{aligned}
\Delta K & =Z_{X} X^{*} e^{2 q_{X} V_{A}} X+Z_{Y} Y^{*} e^{2 q_{Y} V_{A}} Y, \\
\Delta W & =\kappa \frac{X^{k+2} Y}{M_{\text {Planck }}^{k}},
\end{aligned}
$$

where $k=-q_{Y} / q_{X}-2$ is a positive integer, and $\kappa$ can always be chosen to be a real positive constant which is expected to be of order unity since $M_{\text {Planck }}$ is the natural scale to determine the coefficients of higher dimensional operators in the $4 \mathrm{D}$ supergravity superpotential. To determine the vacuum values of $X$ and $Y$, one can integrate out $V_{A}$ and $T_{3}$ to obtain the effective Kähler potential (51), and define the canonically normalized matter fields

$$
\hat{\Phi}_{i}=\sqrt{Z_{i}^{\mathrm{eff}}} \Phi_{i}
$$

while treating $t_{1}$ and $t_{2}$ as a fixed background. Then the effective potential of $\hat{\Phi}_{i}=\{\hat{X}, \hat{Y}\}$ takes the form

$$
\begin{aligned}
V_{\mathrm{eff}} & =m_{X}^{2}|\hat{X}|^{2}+m_{Y}^{2}|\hat{Y}|^{2}+\left(\hat{\kappa} A_{\kappa} \frac{\hat{X}^{k+2} \hat{Y}}{M_{*}^{k}}+\text { h.c. }\right) \\
& +\frac{|\hat{\kappa}|^{2}}{M_{*}^{2 k}}\left(|\hat{X}|^{2 k+4}+(k+2)^{2}|\hat{X}|^{2 k+2}|\hat{Y}|^{2}\right)+\mathcal{O}\left(\frac{m_{3 / 2}^{2}|\hat{\Phi}|^{4}}{M_{G S}^{2}}\right),
\end{aligned}
$$


where $m_{i}(i=X, Y)$ and $A_{\kappa}$ denote the soft SUSY breaking scalar mass and $A$-parameter, respectively, and

$$
\hat{\kappa}=\frac{\kappa}{\sqrt{e^{-(k+3) K_{0}^{\mathrm{eff}} / 3}\left(Z_{X}^{\mathrm{eff}}\right)^{k+2} Z_{Y}^{\mathrm{eff}}}} \simeq \frac{\kappa}{\sqrt{\mathcal{Y}_{X}^{k+2} \mathcal{Y}_{Y}}} .
$$

Note that $\hat{\kappa}=\mathcal{O}(\kappa)$ as $\mathcal{Y}_{i}(i=X, Y)$ are defined to be of order unity, and the scalar potential of the canonically normalized matter fields induced by the Planck-scale-suppressed superpotential is controlled by the winding scale:

$$
M_{*} \sim \frac{M_{\text {Planck }}}{t_{1}^{1 / 2}} \sim t_{1}^{1 / 4} M_{\text {string }} .
$$

As we will see in the next section, in the limit $M_{P Q}^{2} \ll M_{G S}^{2}$, the dominant contribution to $m_{i}$ comes from the $U(1)_{A} D$-term of $\mathcal{O}\left(m_{3 / 2}^{2}\right)$, while $A_{\kappa}$ receives only a suppressed contribution of $\mathcal{O}\left(m_{3 / 2} / 8 \pi^{2}\right)$ from the $F$-components of moduli superfields:

$$
\begin{aligned}
m_{X}^{2} & \simeq-q_{X} g_{A}^{2} D_{A} \simeq-\frac{2 q_{X} \alpha_{3}}{\delta_{G S}} m_{3 / 2}^{2}=\mathcal{O}\left(m_{3 / 2}^{2}\right) \\
m_{Y}^{2} & \simeq-q_{Y} g_{A}^{2} D_{A} \simeq-(k+2) m_{X}^{2} \\
A_{\kappa} & =\mathcal{O}\left(\frac{m_{3 / 2}}{8 \pi^{2}}\right)
\end{aligned}
$$

where we have used $q_{Y}=-(k+2) q_{X}$. A nice feature of the above soft masses is that a tachyonic $m_{X}^{2}$ at tree level can be naturally obtained, which induces a nonzero vacuum value of $X$ at an intermediate scale. One just needs to assume $q_{X} \alpha_{3} / \delta_{G S}>0$, giving $m_{X}^{2}<0$. We then find that $\hat{X}$ and $\hat{Y}$ are stabilized at the following vacuum values:

$$
\begin{aligned}
\langle\hat{X}\rangle & =\left(\frac{m_{X} M_{*}^{k}}{\hat{\kappa} \sqrt{k+2}}\right)^{1 /(k+1)}\left(1+\mathcal{O}\left(\frac{A_{\kappa}^{2}}{m_{3 / 2}^{2}}\right)\right), \\
\langle\hat{Y}\rangle & =\frac{A_{\kappa}}{2(k+2) m_{Y}}\langle\hat{X}\rangle\left(1+\mathcal{O}\left(\frac{\left\langle\hat{X} \hat{X}^{*}\right\rangle}{M_{G S}^{2}}\right)\right),
\end{aligned}
$$

which give

$$
\begin{aligned}
& \langle\hat{X}\rangle \sim M_{P Q} \sim \frac{\left(m_{3 / 2} M_{\text {Planck }}^{k}\right)^{1 /(k+1)}}{t_{1}^{k / 2(k+1)}}, \\
& \langle\hat{Y}\rangle \sim\left(\frac{A_{\kappa}}{m_{Y}}\right)\langle\hat{X}\rangle \sim \mathcal{O}\left(\frac{\langle\hat{X}\rangle}{8 \pi^{2}}\right) .
\end{aligned}
$$

With this result, one can choose appropriate values of model parameters to get an intermediate axion scale in the range $10^{9} \mathrm{GeV} \leq M_{P Q} \leq 10^{12} \mathrm{GeV}$. 
In the above, we have presented a simple model in which the axion scale $M_{P Q}$ is determined by the combined effect of supersymmetry breaking and a Planck-scale suppressed term in the superpotential. For simplicity, we assumed that the axion scale is lower than the $U(1)_{A}$ vector boson mass as

$$
M_{P Q} \ll M_{G S} \sim \delta_{G S} t_{1}^{\frac{3-2 p}{4}} M_{\text {string }},
$$

where $p(=3 / 2$ or 1$)$ denotes the power of $t_{1}$ in the Kähler potential of the MSSM cycle modulus $T_{3}$. On the other hand, in LVS we generically have $M_{\text {string }} / M_{\text {Planck }} \sim 1 / t_{1}^{3 / 4}$ and $m_{3 / 2} / M_{\text {string }} \sim W_{0} / t_{1}^{3 / 4}$, yielding

$$
M_{G S} \sim \delta_{G S} M_{\text {Planck }}\left(\frac{1}{W_{0}} \frac{m_{3 / 2}}{M_{\text {Planck }}}\right)^{p / 3} \sim\left(10^{16}-10^{17}\right) \times\left(\frac{1}{W_{0}} \frac{m_{3 / 2}}{M_{\text {Planck }}}\right)^{p / 3} \mathrm{GeV},
$$

where $W_{0}$ is the flux-induced constant in the effective superpotential. As was noticed in [9], in the presence of loop-induced moduli-mixing, the MSSM gauginos get a mass of $\mathcal{O}\left(m_{3 / 2} / 8 \pi^{2}\right)$, and therefore $m_{3 / 2}$ is required to be in multi-TeV range, e.g. $m_{3 / 2}=\mathcal{O}(10) \mathrm{TeV}$, in order to realize the weak scale SUSY scenario. If we further assume $W_{0} \sim \mathcal{O}(1)$ and $p=3 / 2$, the

resulting $M_{G S}$ is in the range of $10^{9}-10^{10} \mathrm{GeV}$, and therefore might not be high enough to assure the condition $M_{G S} \gg M_{P Q}>10^{9} \mathrm{GeV}$ which has been used throughout our analysis. In such a case, one might need to stabilize the matter fields at a point giving $M_{P Q}$ comparable to or even higher than $M_{G S}$, which would require a separate analysis. In the appendix, we show that it is also possible to stabilize $T_{3}, X$ and $Y$ within the model of (25) and (53) at a point giving $\langle\hat{X}\rangle \sim M_{P Q} \gtrsim M_{G S}$, as long as $\left\langle\hat{X}^{*} \hat{X}\right\rangle \ll M_{G S}^{2} / \delta_{G S}$, and the resulting SUSY breaking patterns are similar to those in the case of $\left\langle\hat{X}^{*} \hat{X}\right\rangle \ll M_{G S}^{2}$. In such situation, $M_{G S}$ corresponds to the QCD axion scale, while $M_{P Q}$ determines the $U(1)_{A}$ gauge boson mass.

\section{SUSY BREAKDOWN AND SOFT TERMS}

We are now ready to discuss SUSY breakdown and the soft terms in the MSSM sector. In the LVS models, the $F$-components of the Kähler moduli $T_{i}(i=1,2,3)$ and the $D$-component of the $U(1)_{A}$ vector superfield $V_{A}$ are the prime candidates for the origin of soft terms [25-27]. If there exist gauge-charged messengers which have a Yukawa coupling to $X$ and/or $Y$, there can be gauge-mediated soft terms arising from the $F$-components of $X$ and/or $Y$ [28]. We first evaluate the vacuum values of these SUSY breaking auxiliary components using the results of the previous two sections. 
From the moduli vacuum values of $T_{1}$ and $T_{2}$ in (12), it is straightforward to find

$$
\begin{aligned}
& \frac{F^{T_{1}}}{t_{1}}=m_{3 / 2}\left[1+\mathcal{O}\left(\frac{\xi_{\alpha^{\prime}}}{a \tilde{t}_{2} t_{1}^{3 / 2}}\right)\right], \\
& \frac{F^{T_{2}}}{\tilde{t}_{2}}=m_{3 / 2}\left[\frac{3}{2 a \tilde{t}_{2}}+\mathcal{O}\left(\frac{1}{\left(a \tilde{t}_{2}\right)^{2}}\right)\right],
\end{aligned}
$$

where the $F$-component of a generic chiral superfield $\Phi^{I}$ is defined as $F^{I}=-e^{K / 2} K^{I \bar{J}}\left(D_{J} W\right)^{*}$, and this expression of $F^{T_{i}}(i=1,2)$ is not affected by the uplifting potential. Combining the vacuum values of the PQ sector fields in (57) with the superfield equation (43), we also find the following vacuum configuration of the $U(1)_{A}$ sector fields:

$$
\begin{aligned}
\left\langle\hat{X}^{*} \hat{X}\right\rangle & \equiv\left\langle Z_{X} X^{*} e^{2 q_{X} V_{A}} X\right\rangle \sim M_{P Q}^{2}, \\
\left\langle\hat{Y}^{*} \hat{Y}\right\rangle & \equiv\left\langle Z_{Y} Y^{*} e^{2 q_{Y} V_{A}} Y\right\rangle \sim \frac{A_{\kappa}^{2} M_{P Q}^{2}}{m_{Y}^{2}} \sim \frac{M_{P Q}^{2}}{\left(8 \pi^{2}\right)^{2}}, \\
\tilde{t}_{3} & =t_{3}-\alpha_{3} \ln t_{1}-\delta_{G S} V_{A}=\mathcal{O}\left(\frac{\delta_{G S} M_{P Q}^{2}}{M_{G S}^{2}}\right), \\
\frac{F^{X}}{X} & \sim A_{\kappa} \sim \frac{m_{3 / 2}}{8 \pi^{2}}\left[1+\mathcal{O}\left(\frac{M_{P Q}^{2}}{M_{G S}^{2}}\right)\right] \\
\frac{F^{Y}}{Y} & \sim \frac{m_{Y}^{2}}{A_{\kappa}} \sim \frac{D_{A}}{A_{\kappa}} \sim 8 \pi^{2} m_{3 / 2}, \\
F^{T_{3}} & =\alpha_{3} \frac{F^{T_{1}}}{t_{1}}+\mathcal{O}\left(\frac{m_{3 / 2} \delta_{G S} M_{P Q}^{2}}{M_{G S}^{2}}\right)=\alpha_{3} m_{3 / 2}+\mathcal{O}\left(\frac{m_{3 / 2} \delta_{G S} M_{P Q}^{2}}{M_{G S}^{2}}\right), \\
g_{A}^{2} D_{A} & =\frac{2 \alpha_{3}}{\delta_{G S}}\left|\frac{F^{T_{1}}}{t_{1}}\right|^{2}+\mathcal{O}\left(\frac{m_{3 / 2}^{2} M_{P Q}^{2}}{M_{G S}^{2}}\right)=\frac{2 \alpha_{3} m_{3 / 2}^{2}}{\delta_{G S}}+\mathcal{O}\left(\frac{m_{3 / 2}^{2} M_{P Q}^{2}}{M_{G S}^{2}}\right),
\end{aligned}
$$

where $m_{Y}^{2}$ denotes the soft scalar mass of $Y$, which is of the order of $D_{A}$. Note that some vacuum values, for instance those of $F^{\Phi_{i}} / \Phi_{i}\left(\Phi_{i}=X, Y\right)$ and the scalar and $F$ components of $V_{A}$, are not invariant under the $T_{3}$-dependent field redefinition (47). More specifically, the original $V_{A}$ in (43) is defined in the Wess-Zumino gauge, and therefore has vanishing scalar and $F$ components. On the other hand, $V_{A}$ in (49) after the field redefinition (477) is defined in the unitary gauge containing the Goldstone superfield $\propto T_{3}+T_{3}^{*}$, and therefore has nonzero scalar and $F$ components coming from $T_{3}+T_{3}^{*}$. As for $F^{\Phi_{i}} / \Phi_{i}\left(\Phi_{i}=X, Y\right)$, the above results denote the vacuum values before the redefinition (47).

Although the above vacuum values of the $U(1)_{A}$ sector fields have been derived in the limit $M_{P Q}^{2} \ll M_{G S}^{2}$, we find (as explained in the appendix) that they remain to be valid even for $M_{P Q}^{2} \gtrsim M_{G S}^{2}$, at least qualitatively, as along as $\tilde{t}_{3} \sim \delta_{G S} M_{P Q}^{2} / M_{G S}^{2} \ll 1$, which would be required for the Kähler potential to be expanded in powers of $\tilde{t}_{3}$ as in (26). 
A notable feature of LVS is the no-scale structure of the large volume modulus $t_{1}$, which leads to a strong suppression of the anomaly mediated contributions. Anomaly mediation is described most conveniently by the super-Weyl-invariant compensator formulation of $4 \mathrm{D}$ SUGRA, involving a chiral compensator superfield $C$ [29]. One can then choose a super-Weyl gauge in which the SUSY breaking (but Poincare-invariant) component of ordinary SUGRA multiplet is vanishing, e.g.

$$
\left.\mathcal{R}\right|_{\theta=\bar{\theta}=0}=0,
$$

where $\mathcal{R}$ is the chiral curvature superfield in $N=1$ superspace. One could also choose the Einstein frame gauge for which $\left.C_{0} \equiv C\right|_{\theta=\bar{\theta}=0}=e^{K / 6}$. Thus the $F$-component of the compensator superfield is given by

$$
\frac{F^{C}}{C_{0}}=m_{3 / 2}+\frac{1}{3} K_{I} F^{I} .
$$

Using the results on the moduli and matter $F$-components, we find

$$
\frac{F^{C}}{C_{0}}=\mathcal{O}\left(\frac{m_{3 / 2} M_{\text {string }}^{2}}{M_{\text {Planck }}^{2}}, \frac{m_{3 / 2} M_{P Q}^{2}}{M_{\text {Planck }}^{2}}\right),
$$

where the piece of $\mathcal{O}\left(m_{3 / 2} M_{P Q}^{2} / M_{\text {Planck }}^{2}\right)$ originates from the $K_{T_{3}} F^{T_{3}}$, while the other piece of $\mathcal{O}\left(m_{3 / 2} M_{\text {string }}^{2} / M_{\text {Planck }}^{2}\right)$ can arise from the mixing between $T_{1}$ and the string dilaton that appears in the $\alpha^{\prime}$ correction to the Kähler potential. This assures that anomaly mediation in LVS can be safely ignored.

With the SUSY breaking auxiliary components given above, we can compute the soft terms of the MSSM gauge and matter multiplets as well as those of the PQ sector matter multiplets. Here we will focus on the soft terms induced by the moduli $F$-components [25] and the $U(1)_{A}$ $D$-component [26, 27], although there can be gauge-mediated soft terms as well. For instance, if there exist gauge-charged messenger fields $\Phi+\Phi^{c}$ with a Yukawa coupling $\propto Y \Phi \Phi^{c}$, gaugino and scalar masses of $\mathcal{O}\left(m_{3 / 2}\right)$ can be induced by $F^{Y} / Y \sim 8 \pi^{2} m_{3 / 2}$ through the conventional gauge mediation mechanism [28], and these gauge-mediated gaugino masses will dominate over the moduli-mediated gaugino masses of $\mathcal{O}\left(m_{3 / 2} / 8 \pi^{2}\right)$. However the presence of such gauge mediation is strongly model dependent. Particularly it depends on whether the model contains exotic matter fields with the right quantum numbers and right couplings. In any case, it is straightforward to incorporate the gauge-mediated soft terms, if there exist any, with the more generic moduli-mediated or $D$-term induced soft terms on which we will concentrate in the following. 
To evaluate the moduli-mediated and $D$-term induced soft parameters, let $T_{I}$ denote the SUSY breaking moduli superfields (not including the compensator $C$ ), and $\Phi_{i}$ denote the visible sector chiral superfields with canonically normalized scalar components $\hat{\phi}_{i}$. For a generic $4 \mathrm{D}$ SUGRA model described by

$$
\begin{aligned}
K & =K_{0}\left(T_{I}, T_{I}^{*}\right)+Z_{i}\left(T_{I}, T_{I}^{*}\right) \Phi_{i}^{*} e^{2 q_{i} V_{A}} \Phi_{i}, \\
W & =\tilde{W}_{0}\left(T_{I}\right)+\frac{1}{3 !} \lambda_{i j k}\left(T_{I}\right) \Phi_{i} \Phi_{j} \Phi_{k}+\frac{1}{n !} \kappa_{i_{1} i_{2} . . i_{n}}\left(T_{I}\right) \Phi_{i_{1}} \Phi_{i_{2}} . . \Phi_{i_{n}}, \\
f_{a} & =f_{a}\left(T_{I}\right),
\end{aligned}
$$

soft SUSY breaking terms of canonically normalized components fields take the form

$$
\begin{aligned}
\mathcal{L}_{\text {soft }}= & -\frac{1}{2} M_{a} \lambda^{a} \lambda^{a}-\frac{1}{2} m_{i}^{2}\left|\hat{\phi}_{i}\right|^{2}-\frac{1}{3 !} A_{i j k} \hat{\lambda}_{i j k} \hat{\phi}_{i} \hat{\phi}_{j} \hat{\phi}_{k} \\
& -\frac{1}{n !} A_{\kappa}^{i_{1} i_{2} . . i_{n}} \hat{\kappa}_{i_{1} i_{2} . . i_{n}} \hat{\phi}_{i_{1}} \hat{\phi}_{i_{2} .} . \hat{\phi}_{i_{n}}+\text { h.c. },
\end{aligned}
$$

where $\hat{\lambda}_{i j k}$ and $\hat{\kappa}_{i_{1} i_{2} . i_{n}}$ denote the canonically normalized Yukawa couplings,

$$
\begin{aligned}
\hat{\lambda}_{i j k} & =\frac{\lambda_{i j k}}{\sqrt{e^{-K_{0}} Z_{i} Z_{j} Z_{k}}}, \\
\hat{\kappa}_{i_{1} i_{2} . . i_{n}} & =\frac{\kappa_{i_{1} i_{2} . . i_{n}}}{\sqrt{e^{-n K_{0} / 3} Z_{i_{1}} Z_{i_{2} . .} Z_{i_{n}}}},
\end{aligned}
$$

and the soft SUSY breaking parameters (at scales around the cutoff scale) are then given by

$$
\begin{aligned}
A_{i j k} & =-F^{I} \partial_{I} \ln \left(\frac{\lambda_{i j k}}{e^{-K_{0}} Z_{i} Z_{j} Z_{k}}\right), \\
A_{\kappa}^{i_{1} i_{2} . . i_{n}} & =(n-3) \frac{F^{C}}{C_{0}}-F^{I} \partial_{I} \ln \left(\frac{\kappa_{i_{1} i_{2 . . .}}}{e^{-n K_{0} / 3} Z_{i_{1}} Z_{i_{2} . .} Z_{i_{n}}}\right), \\
m_{i}^{2} & =\frac{2}{3} V_{F}-F^{I} F^{\bar{J}} \partial_{I} \partial_{\bar{J}} \ln \left(e^{-K_{0} / 3} Z_{i}\right)-\left(q_{i}+\eta^{I} \partial_{I} \ln Z_{i}\right) g_{A}^{2} D_{A} \\
\frac{M_{a}}{g_{a}^{2}} & =\frac{1}{2} F^{I} \partial_{I} f_{a}-\frac{1}{8 \pi^{2}} \sum_{i} \operatorname{Tr}\left(T_{a}^{2}\left(\Phi_{i}\right)\right) F^{I} \partial_{I} \ln \left(e^{-K_{0} / 3} Z_{i}\right)+\frac{b_{a}}{16 \pi^{2}} \frac{F^{C}}{C_{0}},
\end{aligned}
$$

where $V_{F}=K_{I \bar{J}} F^{I} F^{\bar{J}}-3 m_{3 / 2}^{2}$ is the $F$-term scalar potential. Here we consider the tree level contributions (in the sense of $4 \mathrm{D}$ effective SUGRA) to $m_{i}$ and $A$-parameters as they provide the dominant part at the cutoff scale. On the other hand, we included the full 1-loop contributions to gaugino masses [30], which can be relevant for the gaugino masses derived from the effective theory after the massive $U(1)_{A}$ vector multiplet (including $T_{3}$ ) is integrated out.

The above expression of soft masses can be applied to the LVS model (25) with the PQ sector given by (53). We find

$$
m_{i}^{2} \simeq-q_{i} g_{A}^{2} D_{A}=\left[-\frac{2 q_{i} \alpha_{3}}{\delta_{G S}}+\mathcal{O}\left(\frac{M_{P Q}^{2}}{M_{G S}^{2}}\right)\right] m_{3 / 2}^{2}=\mathcal{O}\left(m_{3 / 2}^{2}\right)
$$




$$
\begin{aligned}
& A_{i j k} \simeq\left[\left(\frac{3}{2 a}-\beta_{2}\right) \partial_{\hat{t}_{2}} \ln \mathcal{Y}_{i} \mathcal{Y}_{j} \mathcal{Y}_{k}+\left(\alpha_{3}-\beta_{3}\right) \partial_{\hat{t}_{3}} \ln \mathcal{Y}_{i} \mathcal{Y}_{j} \mathcal{Y}_{k}\right] m_{3 / 2}=\mathcal{O}\left(\frac{m_{3 / 2}}{8 \pi^{2}}\right) \\
& A_{\kappa} \simeq\left[\left(\frac{3}{2 a}-\beta_{2}\right) \partial_{\hat{t}_{2}} \ln \left(\mathcal{Y}_{X}^{k+2} \mathcal{Y}_{Y}\right)+\left(\alpha_{3}-\beta_{3}\right) \partial_{\hat{t}_{3}} \ln \left(\mathcal{Y}_{X}^{k+2} \mathcal{Y}_{Y}\right)\right] m_{3 / 2}=\mathcal{O}\left(\frac{m_{3 / 2}}{8 \pi^{2}}\right) \\
& \frac{M_{a}}{g_{a}^{2}} \simeq \frac{k_{a}}{2} F^{T_{3}}=\left[\frac{\alpha_{3} k_{a}}{2}+\mathcal{O}\left(\frac{\delta_{G S} M_{P Q}^{2}}{M_{G S}^{2}}\right)\right] m_{3 / 2}=\mathcal{O}\left(\frac{m_{3 / 2}}{8 \pi^{2}}\right),
\end{aligned}
$$

where we have kept only the dominant part in the limit $M_{P Q}^{2} \ll M_{G S}^{2}$. Here the matter Kähler metric is given by $Z_{i}=\mathcal{Y}_{i}\left(\hat{t}_{2}, \hat{t}_{3}\right) / t_{1}$, and we assumed that $\mathcal{Y}_{i}$ is a generic function of $\hat{t}_{2}=t_{2}-\beta_{2} \ln t_{1}$ and $\hat{t}_{3}=t_{3}-\beta_{3} \ln t_{1}-\delta_{G S} V_{A}$, with $\partial_{a} \ln \mathcal{Y}_{i}=\mathcal{O}(1)\left(a=\hat{t}_{2}, \hat{t}_{3}\right)$.

The most notable feature of the above soft masses is the relative enhancement of the scalar masses compared to the gaugino masses. Gaugino masses and $A$-parameters induced by the moduli $F$-components are generically of $\mathcal{O}\left(m_{3 / 2} / 8 \pi^{2}\right)$, while sfermion masses induced by the $U(1)_{A} D$-term are of $\mathcal{O}\left(m_{3 / 2}\right)$. Note that loop-induced moduli mixing, particularly the one described by $\alpha_{3}$, is crucial for the soft masses comparable to $m_{3 / 2}$ or $m_{3 / 2} / 8 \pi^{2}$. An intriguing feature of our results is that the $D$-term induced sfermion masses are $\mathcal{O}\left(m_{3 / 2}\right)$, although they result from a loop-induced moduli mixing. This is due to the suppression of the $U(1)_{A}$ gauge boson mass-square by $\delta_{G S}^{2}$. Since the $D$-term contribution to sfermion masses arises from the exchange of the $U(1)_{A}$ gauge boson, the loop-suppression factor $\alpha_{3}$ is compensated by the enhancement factor $1 / \delta_{G S}$ in $1 / M_{A}^{2}$, and this makes the $D$-term contribution to be of $\mathcal{O}\left(m_{3 / 2}^{2}\right)$.

In the case $M_{P Q}^{2} \ll M_{G S}^{2}$, the above soft parameters can be obtained also from the effective theory constructed by integrating out the massive $V_{A}$ and $T_{3}$. Indeed, by applying (68) to the effective theory described by the effective Kähler potential (51) and the effective gauge kinetic function (48), we find the same result up to small corrections suppressed by $M_{P Q}^{2} / M_{G S}^{2}$ :

$$
\begin{aligned}
m_{i}^{2} & =-\left|F^{T_{1}}\right|^{2} \partial_{T_{1}} \partial_{T_{1}^{*}} \ln \left(e^{-K_{0}^{\mathrm{eff}} / 3} Z_{i}^{\mathrm{eff}}\right)=-\frac{2 q_{i} \alpha_{3}}{\delta_{G S}} m_{3 / 2}^{2} \\
A_{i j k} & =\left[\frac{3}{2 a} \partial_{t_{2}} \ln \mathcal{Y}_{i}^{\mathrm{eff}} \mathcal{Y}_{j}^{\mathrm{eff}} \mathcal{Y}_{k}^{\mathrm{eff}}+\partial_{t_{1}} \ln \mathcal{Y}_{i}^{\mathrm{eff}} \mathcal{Y}_{j}^{\mathrm{eff}} \mathcal{Y}_{k}^{\mathrm{eff}}\right] m_{3 / 2}, \\
A_{\kappa} & =\left[\frac{3}{2 a} \partial_{t_{2}} \ln \left(\left(\mathcal{Y}_{X}^{\mathrm{eff}}\right)^{k+2} \mathcal{Y}_{Y}^{\mathrm{eff}}\right)+t_{1} \partial_{t_{1}} \ln \left(\left(\mathcal{Y}_{X}^{\mathrm{eff}}\right)^{k+2} \mathcal{Y}_{Y}^{\mathrm{eff}}\right)\right] m_{3 / 2}, \\
\frac{M_{a}}{g_{a}^{2}} & =-\frac{1}{8 \pi^{2}} \sum_{i} \operatorname{Tr}\left(T_{a}^{2}\left(\Phi_{i}\right)\right) F^{T_{1}} \partial_{t_{1}} \ln \left(e^{-K_{0}^{\mathrm{eff}} / 3} Z_{i}^{\mathrm{eff}}\right)=\frac{\alpha_{3} k_{a}}{2} m_{3 / 2},
\end{aligned}
$$

where

$$
Z_{i}^{\mathrm{eff}}\left(t_{1}, t_{2}\right)=\frac{Z_{i}\left(t_{1}, \hat{t}_{2}=t_{2}-\beta_{2} \ln t_{1}, \hat{t}_{3}=\left(\alpha_{3}-\beta_{3}\right) \ln t_{1}\right)}{t_{1}^{2 q_{i} \alpha_{3} / \delta_{G S}}}
$$




$$
\begin{aligned}
& =\frac{\mathcal{Y}_{i}\left(\hat{t}_{2}=t_{2}-\beta_{2} \ln t_{1}, \hat{t}_{3}=\left(\alpha_{3}-\beta_{3}\right) \ln t_{1}\right)}{t_{1}^{1+2 q_{i} \alpha_{3} / \delta_{G S}}} \\
& \equiv \frac{\mathcal{Y}_{i}^{\mathrm{eff}}\left(t_{1}, t_{2}\right)}{t_{1}^{1+2 q_{i} \alpha_{3} / \delta_{G S}}},
\end{aligned}
$$

and we have used the anomaly matching condition (40) for the gaugino masses.

\section{CONCLUSIONS}

As we have seen, the LVS scenario [7] leads to a somewhat different pattern of soft terms than the conventional KKLT scenario [3]. While in the LVS case we find soft scalar masses of the order of the gravitino mass (due to the $D$-term contribution), the KKLT scenario leads to suppressed scalar masses of order $m_{3 / 2} / \ln \left(M_{\text {Planck }} / m_{3 / 2}\right)$ as expected in mirage mediation [5, 6]. The moduli-mediated gaugino masses and $A$-terms appear to be of order $m_{3 / 2} / \ln \left(M_{\text {Planck }} / m_{3 / 2}\right)$ in both cases. Therefore, unless there exist more model dependent gauge-mediated contributions dominating over the moduli mediation, soft masses in LVS with moduli-mixing shows a loop-hierarchy pattern: $m_{i} \sim 8 \pi^{2} M_{a}$. Another key difference between LVS and KKLT is the relative importance of anomaly mediation. In the LVS, due to the no-scale structure, anomaly mediation is negligible, while in the KKLT scenario it becomes comparable to the moduli-mediation, leading to the mirage unification of soft masses at a scale different from $M_{G U T} \sim 2 \times 10^{16} \mathrm{GeV}[6]$.

The two set-ups differ also in the way to obtain a small value of $m_{3 / 2}$. In the KKLT scenario this comes from a small value of the superpotential $W$, while in the LVS models one assumes $W \sim 1$ and a small $m_{3 / 2}$ is the result of the large volume suppression. Large volume, of course,

implies a large hierarchy between the Planck scale and the string scale $M_{\text {Planck }}^{2} / M_{\text {string }}^{2} \sim \mathcal{V}_{C Y}$. The natural setting for LVS would be a string scale at an intermediate value of approximately $10^{11} \mathrm{GeV}$ and a gravitino mass as well as the soft mass terms at the (multi) TeV-scale. In the KKLT set-up the natural value of $M_{\text {string }}$ would be rather large, somewhere between the Planck scale and a possible GUT scale at $10^{16} \mathrm{GeV}$. The two scenarii can be connected in principle by changing the value of the superpotential. With the results for the soft terms in the presence of loop-induced moduli mixing, assuming soft masses to be in the $\mathrm{TeV}$ range, it is likely that a gravitino mass much heavier than $\mathcal{O}\left(8 \pi^{2}\right)$ TeV requires a severe fine tuning of Kähler potential. For the LVS case (assuming $W \sim 1$ ) this would imply the string scale is around $10^{11} \mathrm{GeV}$, which is quite small compared to the GUT scale of $10^{16} \mathrm{GeV}$. In that sense, the pure LVS- 
scenario might be difficult to be compatible with gauge coupling unification at $10^{16} \mathrm{GeV}$. Such a GUT-picture would require a value of the superpotential that is small compared to one.

Our considerations are valid for the simple set-up described and we have focused on the generic contributions to soft terms from the moduli $F$-components and the $U(1)_{A} D$-term. Other more model dependent contributions could be present as well. For example, the fields $X$ and $Y$ introduced in section 3 to break the global Peccei-Quinn symmetry at an intermediate scale could act as the origin of additional "gauge mediated" contributions. It is straightforward to incorporate those gauge mediated contributions with the soft terms discussed in this paper. This, in connection with the incorporation of a QCD axion, will be discussed in detail in a future publication [31].

\section{Acknowledgments}

KC and CSS are supported by the KRF Grants funded by the Korean Government (KRF2008-314-C00064 and KRF-2007-341-C00010) and the KOSEF Grant funded by the Korean Government (No. 2009-0080844). HPN and MT are supported by the SFB-Tansregio TR33

"The Dark Universe" (Deutsche Forschungsgemeinschaft) and the European Union 7th network program "Unification in the LHC era" (PITN-GA-2009-237920).

\section{Appendix A}

In section 3, we have discussed the stabilization of the $D$-flat direction based on the effective theory constructed in the limit $M_{P Q}^{2} \ll M_{G S}^{2}$. Here we provide an analysis of the stabilization of the $U(1)_{A}$ sector in more general situation including the case $M_{P Q} \sim M_{G S}$.

Under the assumption that $\tilde{t}_{3}=t_{3}-\alpha_{3} \ln t_{1}-\delta_{G S} V_{A}$ has a small vacuum value, which would be fulfilled if $M_{P Q}^{2} \ll M_{G S}^{2} / \delta_{G S}$, the Käher potential and superpotential of the model are given by

$$
\begin{aligned}
K= & -3 \ln t_{1}+\frac{2\left(\tilde{t}_{2}^{3 / 2}-\xi_{\alpha^{\prime}}\right)}{t_{1}^{3 / 2}}+\frac{1}{2 t_{1}^{p}}\left(\tilde{t}_{3}^{2}+\mathcal{O}\left(\widetilde{t}_{3}^{3}\right)\right) \\
& +Z_{X} X^{*} e^{2 q_{X} V_{A}} X+Z_{Y} Y^{*} e^{2 q_{Y} V_{A}} Y \\
W= & W_{0}+A e^{-a T_{2}}+\kappa \frac{X^{k+2} Y}{M_{\text {Planck }}^{k}}
\end{aligned}
$$

where $Z_{i}=\mathcal{Y}_{i}\left(\hat{t}_{2}, \hat{t}_{3}\right) / t_{1}(i=X, Y)$. Inserting the values of $t_{1,2}$ and $F^{T_{1,2}}$ obtained in (12) and 
(61) into the SUGRA scalar potential:

$$
V_{\mathrm{SUGRA}}=e^{K}\left\{K^{I \bar{J}} D_{I} W\left(D_{J} W\right)^{*}-3|W|^{2}\right\}+\frac{1}{2} g_{A}^{2} D_{A}^{2},
$$

we find the following scalar potential of $t_{3}, X$ and $Y$ :

$$
\begin{aligned}
V\left(t_{3}, X, Y\right)= & \frac{g_{A}^{2}}{2}\left(M_{G S}^{2} \frac{2 \tilde{t}_{3}}{\delta_{G S}}-q_{X} \tilde{Z}_{X}|X|^{2}-q_{Y} \tilde{Z}_{Y}|Y|^{2}\right)^{2} \\
& -\left(\frac{4 m_{3 / 2}^{2} M_{G S}^{2}}{\delta_{G S}^{2}}\right)\left(\alpha_{3} \tilde{t}_{3}+\frac{(2-p)(p-1)^{2}}{2} \tilde{t}_{3}^{2}\right) \\
& -m_{3 / 2}^{2}\left(\beta_{a} \partial_{a} \ln \mathcal{Y}_{X}+\left(\gamma_{a} \gamma_{b} \partial_{a} \partial_{b} \ln \mathcal{Y}_{X}\right)\right) Z_{X}|X|^{2} \\
& -m_{3 / 2}^{2}\left(\beta_{a} \partial_{a} \ln \mathcal{Y}_{Y}+\left(\gamma_{a} \gamma_{b} \partial_{a} \partial_{b} \ln \mathcal{Y}_{Y}\right)\right) Z_{Y}|Y|^{2} \\
& +\left(A_{\kappa} \frac{\kappa X^{k+2} Y}{t_{1}^{3 / 2}}+\text { h.c. }\right)+\frac{|\kappa|^{2}}{t_{1}^{3}}\left(\frac{\left|(k+2) X^{k+1} Y\right|^{2}}{Z_{X}}+\frac{\left|X^{k+2}\right|^{2}}{Z_{Y}}\right),
\end{aligned}
$$

where

$$
\begin{aligned}
q_{i} \tilde{Z}_{i} & =\left(q_{i}-\frac{\delta_{G S}}{2} \partial_{\hat{t}_{3}}\right) Z_{i}=q_{i} Z_{i}-\frac{\delta_{G S}}{2} \partial_{\hat{t}_{3}} Z_{i}, \\
\beta_{a} \partial_{a} & =\beta_{2} \partial_{\hat{t}_{2}}+\beta_{3} \partial_{\hat{t}_{3}}, \\
\gamma_{a} \partial_{a} & =\left(\frac{3}{2 a}-\beta_{2}\right) \partial_{\hat{t}_{2}}+\left(\alpha_{3}-\beta_{3}+(p-1) \tilde{t}_{3}\right) \partial_{\hat{t}_{3}}, \\
A_{\kappa} & =\left(\gamma_{a} \partial_{a} \ln \left(\mathcal{Y}_{X}^{k+2} \mathcal{Y}_{Y}\right)\right) m_{3 / 2} .
\end{aligned}
$$

From the SUGRA expression of the $F$-component, i.e. $F^{I}=-e^{K / 2} K^{I \bar{J}}\left(D_{J} W\right)^{*}$, we find also

$$
\begin{aligned}
F^{T_{3}} & =\left(\alpha_{3}+(p-1) \tilde{t}_{3}\right) m_{3 / 2} \\
\frac{F^{\Phi_{i}}}{\Phi_{i}} & =-\left(\gamma_{a} \partial_{a} \ln \mathcal{Y}_{i}\right) m_{3 / 2}-\frac{\left(\partial_{\Phi_{i}} W_{\mathrm{m}}\right)^{*}}{t_{1}^{3 / 2} Z_{i} \Phi_{i}} \quad\left(\Phi_{i}=X, Y\right),
\end{aligned}
$$

where $W_{\mathrm{m}}=\kappa X^{k+2} Y / M_{\text {Planck }}^{k}$. The stationary point of the potential (A2) is given by

$$
\begin{aligned}
\tilde{t}_{3} & =\frac{\delta_{G S} M_{P Q}^{2}}{M_{G S}^{2}}\left(1+\mathcal{O}\left(\delta_{G S}\right)\right), \\
|\hat{X}| & =\sqrt{Z_{X}|X|^{2}}=\left(\sqrt{\frac{q_{X} g_{A}^{2} D_{A}}{k+2}} \frac{M_{*}^{k}}{|\hat{\kappa}|}\right)^{1 /(k+1)}\left(1+\mathcal{O}\left(\frac{A_{\kappa}^{2}}{D_{A}}\right)\right), \\
|\hat{Y}| & =\sqrt{Z_{Y}|Y|^{2}}=\frac{\left|A_{\kappa} \hat{X}\right|}{2 \sqrt{q_{X} g_{A}^{2} D_{A}(k+2)^{3}}}\left(1+\mathcal{O}\left(\frac{A_{\kappa}^{2}}{D_{A}}\right)\right),
\end{aligned}
$$


where

$$
\begin{aligned}
M_{*} & =\frac{M_{\text {Planck }}}{t_{1}^{1 / 2} \sim t_{1}^{1 / 4} M_{\text {string }},} \\
M_{P Q}^{2} & =\left(q_{X}-\frac{\delta_{G S}}{2} \partial_{\hat{t}_{3}}\right)^{2} Z_{X}|X|^{2}+\left(q_{X}-\frac{\delta_{G S}}{2} \partial_{\hat{t}_{3}}\right)^{2} Z_{Y}|Y|^{2}, \\
g_{A}^{2} D_{A} & =g_{A}^{2}\left(\frac{2 M_{G S}^{2} \tilde{t}_{3}}{\delta_{G S}}-q_{i} \tilde{Z}_{i}\left|\Phi_{i}\right|^{2}\right)=\left(\frac{2 q_{X} \alpha_{3}}{\delta_{G S}}+(2-p)(p-1) \frac{M_{P Q}^{2}}{M_{G S}^{2}}\right) \frac{m_{3 / 2}^{2}}{q_{X}} .
\end{aligned}
$$

To see that this stationary point is a stable (local) minimum, we can compute the mass-square eigenvalues $m_{1,2}^{2}$ of the $D$-flat directions. We then find

$$
\begin{aligned}
& m_{1}^{2}=\left(\frac{\left.4(k+1)\left(\frac{2 q_{X} \alpha_{3}}{\delta_{G S}}\right)+k \frac{M_{P Q}^{2}}{M_{G S}^{2}}\right) m_{3 / 2}^{2}}{1+\frac{M_{P Q}^{2}}{M_{G S}^{2}}}\right) \\
& m_{2}^{2}=-2 q_{Y} g_{A}^{2} D_{A}=2(k+2)\left(\frac{2 q_{X} \alpha_{3}}{\delta_{G S}}+(2-p)(p-1) \frac{M_{P Q}^{2}}{M_{G S}^{2}}\right) m_{3 / 2}^{2}
\end{aligned}
$$

showing that both eigenvalues are positive, so the solution (A5) is indeed a stable (local) minimum. We can now make an order of magnitude estimate for the vacuum configuration of the $U(1)_{A}$ sector fields:

$$
\begin{aligned}
D_{A} & \sim\left(\frac{\alpha_{3}}{\delta_{G S}}+\frac{(p-1) M_{P Q}^{2}}{M_{G S}^{2}}\right) m_{3 / 2}^{2}, \\
F^{T_{3}} & \sim\left(\alpha_{3}+\frac{(p-1) \delta_{G S} M_{P Q}^{2}}{M_{G S}^{2}}\right) m_{3 / 2}, \\
A_{\kappa} & \sim\left(\frac{1}{8 \pi^{2}}+\frac{(p-1) \delta_{G S} M_{P Q}^{2}}{M_{G S}^{2}}\right) m_{3 / 2} \\
|\hat{X}| & \sim M_{P Q} \sim\left(\sqrt{D_{A}} M_{*}^{k}\right)^{1 /(k+1)}, \quad|\hat{Y}| \sim \frac{A_{\kappa}}{\sqrt{D_{A}}}|\hat{X}|, \\
\frac{F^{X}}{X} & \sim\left(\gamma_{a} \partial_{a} \ln \mathcal{Y}_{X}\right) m_{3 / 2} \sim A_{\kappa}, \quad \frac{F^{Y}}{Y} \sim \frac{D_{A}}{A_{\kappa}},
\end{aligned}
$$

where $\delta_{G S} \sim \alpha_{3} \sim 1 / 8 \pi^{2}$, and we have ignored the coefficients of order unity in the expression. Note that in the limit $M_{P Q}^{2} \ll M_{G S}^{2}$, the above results reproduce (57) and (62) obtained in section 4 based on the effective theory constructed by integrating out the massive $U(1)_{A}$ vector superfield in the limit $M_{P Q}^{2} \ll M_{G S}^{2}$. The above results show also that (57) and (62) are valid even when $M_{P Q}^{2} \gtrsim M_{G S}^{2}$, at least qualitatively, as along as $\tilde{t}_{3} \sim \delta_{G S} M_{P Q}^{2} / M_{G S}^{2} \ll 1$, which is 
required for the Kähler potential to have a meaningful expansion in powers of $\tilde{t}_{3}$ as in (A1).

[1] J. P. Derendinger, L. E. Ibanez and H. P. Nilles, "On the low-energy D = 4, N=1 supergravity theory extracted from the D = 10, N=1 superstring," Phys. Lett. B 155, 65 (1985); Nucl. Phys. B 267, 365 (1986); M. Dine, R. Rohm, N. Seiberg and E. Witten, "Gluino condensation in superstring models," Phys. Lett. B 156, 55 (1985).

[2] S. B. Giddings, S. Kachru and J. Polchinski, "Hierarchies from fluxes in string compactifications," Phys. Rev. D 66, 106006 (2002) arXiv:hep-th/0105097; K. Dasgupta, G. Rajesh and S. Sethi, "M theory, orientifolds and G-flux," JHEP 9908, 023 (1999) arXiv:hep-th/9908088.

[3] S. Kachru, R. Kallosh, A. Linde and S. P. Trivedi, "de Sitter vacua in string theory," Phys. Rev. D 68, 046005 (2003) arXiv:hep-th/0301240.

[4] K. Choi, A. Falkowski, H. P. Nilles, M. Olechowski and S. Pokorski, "Stability of flux compactifications and the pattern of supersymmetry breaking," JHEP 0411, 076 (2004) arXiv:hep-th/0411066].

[5] K. Choi, A. Falkowski, H. P. Nilles and M. Olechowski, "Soft supersymmetry breaking in KKLT flux compactification," Nucl. Phys. B 718, 113 (2005) arXiv:hep-th/0503216.

[6] K. Choi, K. S. Jeong and K. i. Okumura, "Phenomenology of mixed modulus-anomaly mediation in fluxed string compactifications and brane models," JHEP 0509, 039 (2005) arXiv:hep-ph/0504037; M. Endo, M. Yamaguchi and K. Yoshioka, "A bottom-up approach to moduli dynamics in heavy gravitino scenario: Superpotential, soft terms and sparticle mass spectrum," Phys. Rev. D 72, 015004 (2005) arXiv:hep-ph/0504036]; A. Falkowski, O. Lebedev and Y. Mambrini, "SUSY phenomenology of KKLT flux compactifications," JHEP 0511, 034 (2005) arXiv:hep-ph/0507110; O. Loaiza-Brito, J. Martin, H. P. Nilles and M. Ratz, " $\log (\mathrm{M}(\mathrm{Pl} / \mathrm{m}(3 / 2)))$," AIP Conf. Proc. 805, 198 (2006) arXiv:hep-th/0509158.

[7] V. Balasubramanian, P. Berglund, J. P. Conlon and F. Quevedo, "Systematics of moduli stabilisation in Calabi-Yau flux compactifications," JHEP 0503, 007 (2005) arXiv:hep-th/0502058; J. P. Conlon, F. Quevedo and K. Suruliz, "Large-volume flux compactifications: Moduli spectrum and D3/D7 soft supersymmetry breaking," JHEP 0508, 007 (2005) arXiv:hep-th/0505076.

[8] R. Blumenhagen, J. P. Conlon, S. Krippendorf, S. Moster and F. Quevedo, "SUSY breaking in local string/F-theory models," JHEP 0909, 007 (2009) arXiv:0906.3297 [hep-th]]. 
[9] J. P. Conlon and F. G. Pedro, "Moduli redefinitions and moduli stabilisation," JHEP 1006, 082 (2010) arXiv:1003.0388 [hep-th]].

[10] L. E. Ibanez and H. P. Nilles, "Low-energy remnants of superstring anomaly cancellation terms," Phys. Lett. B 169, 354 (1986); K. Choi, "Supersymmetry breaking for the observable sector in superstring models," Z. Phys. C 39, 219 (1988).

[11] H. P. Nilles, M. Olechowski and M. Yamaguchi, "Supersymmetry breaking and soft terms in Mtheory," Phys. Lett. B 415, 24 (1997) arXiv:hep-th/9707143; H. P. Nilles, M. Olechowski and M. Yamaguchi, "Supersymmetry breakdown at a hidden wall," Nucl. Phys. B 530, 43 (1998) arXiv:hep-th/9801030]; A. Lukas, B. A. Ovrut and D. Waldram, "On the four-dimensional effective action of strongly coupled heterotic string theory," Nucl. Phys. B 532, 43 (1998) arXiv:hep-th/9710208]; K. Choi, H. B. Kim and C. Munoz, "Four-dimensional effective supergravity and soft terms in M-theory," Phys. Rev. D 57, 7521 (1998) arXiv:hep-th/9711158.

[12] R. Blumenhagen, S. Moster and E. Plauschinn, "Moduli stabilisation versus chirality for MSSM like Type IIB orientifolds," JHEP 0801, 058 (2008) [arXiv:0711.3389 [hep-th]].

[13] J. P. Derendinger, S. Ferrara, C. Kounnas and F. Zwirner, "On loop corrections to string effective field theories: Field dependent gauge couplings and sigma model anomalies," Nucl. Phys. B 372, $145(1992)$.

[14] L. J. Dixon, V. Kaplunovsky and J. Louis, "Moduli dependence of string loop corrections to gauge coupling constants," Nucl. Phys. B 355, 649 (1991).

[15] K. Choi, J. E. Kim and H. P. Nilles, "Cosmological constant and soft terms in supergravity," Phys. Rev. Lett. 73, 1758 (1994) arXiv:hep-ph/9404311].

[16] M. Dine, N. Seiberg and E. Witten, "Fayet-Iliopoulos terms in string theory," Nucl. Phys. B 289, 589 (1987); M. Dine, I. Ichinose and N. Seiberg, "F terms and D terms in string theory," Nucl. Phys. B 293, 253 (1987).

[17] M. B. Green and J. H. Schwarz, "Anomaly cancellation in supersymmetric D=10 gauge theory and superstring Theory," Phys. Lett. B 149, 117 (1984).

[18] R. D. Peccei and H. R. Quinn, "Constraints imposed by CP conservation in the presence of instantons," Phys. Rev. D 16, 1791 (1977); "CP conservation in the presence of instantons," Phys. Rev. Lett. 38, 1440 (1977).

[19] N. Arkani-Hamed, M. Dine and S. P. Martin, "Dynamical supersymmetry breaking in models with a Green-Schwarz mechanism," Phys. Lett. B 431, 329 (1998) arXiv:hep-ph/9803432]; T. Bar- 
reiro, B. de Carlos, J. A. Casas and J. M. Moreno, "Anomalous U(1), gaugino condensation and supergravity," Phys. Lett. B 445, 82 (1998) arXiv:hep-ph/9808244.

[20] K. Choi and K. S. Jeong, "Supersymmetry breaking and moduli stabilization with anomalous U(1) gauge symmetry," JHEP 0608, 007 (2006) arXiv:hep-th/0605108].

[21] S. Weinberg, "A new light boson?," Phys. Rev. Lett. 40, 223 (1978); F. Wilczek, "Problem of strong P and T invariance in the presence of instantons," Phys. Rev. Lett. 40, 279 (1978).

[22] J. E. Kim, "Weak interaction singlet and strong CP invariance," Phys. Rev. Lett. 43, 103 (1979); M. A. Shifman, A. I. Vainshtein and V. I. Zakharov, "Can confinement ensure natural CP invariance of strong interactions?," Nucl. Phys. B 166, 493 (1980); M. Dine, W. Fischler and M. Srednicki, "A simple solution to the strong CP problem with a harmless axion," Phys. Lett. B 104, 199 (1981); A. R. Zhitnitsky, "On possible suppression of the axion hadron interactions. (in Russian)," Sov. J. Nucl. Phys. 31, 260 (1980) [Yad. Fiz. 31, 497 (1980)].

[23] For a recent review on axions and the strong CP problem, see J. E. Kim and G. Carosi, "Axions and the strong CP problem," arXiv:0807.3125 [hep-ph].

[24] H. Murayama, H. Suzuki and T. Yanagida, "Radiative breaking of Peccei-Quinn symmetry at the intermediate mass scale," Phys. Lett. B 291, 418 (1992); K. Choi, E. J. Chun and J. E. Kim, "Cosmological implications of radiatively generated axion scale," Phys. Lett. B 403, 209 (1997) arXiv:hep-ph/9608222.

[25] V. S. Kaplunovsky and J. Louis, "Model independent analysis of soft terms in effective supergravity and in Phys. Lett. B 306, 269 (1993) arXiv:hep-th/9303040; A. Brignole, L. E. Ibanez and C. Munoz, "Towards a theory of soft terms for the supersymmetric Standard Model," Nucl. Phys. B 422, 125 (1994) [Erratum-ibid. B 436, 747 (1995)] [arXiv:hep-ph/9308271].

[26] Y. Kawamura and T. Kobayashi, "Soft scalar masses in string models with anomalous $U(1)$ symmetry," Phys. Lett. B 375, 141 (1996) [Erratum-ibid. B 388, 867 (1996)] arXiv:hep-ph/9601365]; P. Binetruy and E. Dudas, "Gaugino condensation and the anomalous U(1)," Phys. Lett. B 389, 503 (1996) arXiv:hep-th/9607172]; G. R. Dvali and A. Pomarol, "Anomalous U(1) as a mediator of supersymmetry breaking," Phys. Rev. Lett. 77, 3728 (1996) arXiv:hep-ph/9607383.

[27] T. Higaki, Y. Kawamura, T. Kobayashi and H. Nakano, "Anomalous U(1) D-term contribution in type I string models," Phys. Rev. D 69, 086004 (2004) arXiv:hep-ph/0308110; B. Kors and P. Nath, "Hierarchically split supersymmetry with Fayet-Iliopoulos D-terms in string theory," Nucl. Phys. B 711, 112 (2005) arXiv:hep-th/0411201]; K. S. Babu, T. Enkhbat and 
B. Mukhopadhyaya, "Split supersymmetry from anomalous U(1)," Nucl. Phys. B 720, 47 (2005) arXiv:hep-ph/0501079]; E. Dudas and S. K. Vempati, "Large D-terms, hierarchical soft spectra and moduli stabilisation," Nucl. Phys. B 727, 139 (2005) arXiv:hep-th/0506172 ; C. A. Scrucca, "Soft masses in superstring models with anomalous U(1) symmetries," JHEP 0712, 092 (2007) arXiv:0710.5105 [hep-th]]; E. Dudas, Y. Mambrini, S. Pokorski, A. Romagnoni and M. Trapletti, "Gauge vs. Gravity mediation in models with anomalous U(1)'s," JHEP 0903, 011 (2009) arXiv:0809.5064 [hep-th]].

[28] M. Dine, W. Fischler and M. Srednicki, "Supersymmetric technicolor," Nucl. Phys. B 189, 575 (1981); S. Dimopoulos and S. Raby, "Supercolor," Nucl. Phys. B 192, 353 (1981); M. Dine and W. Fischler, "A phenomenological model of particle physics based on supersymmetry," Phys. Lett. B 110, 227 (1982); M. Dine and A. E. Nelson, "Dynamical supersymmetry breaking at low-energies," Phys. Rev. D 48, 1277 (1993) [arXiv:hep-ph/9303230; M. Dine, A. E. Nelson and Y. Shirman, "Low-energy dynamical supersymmetry breaking simplified," Phys. Rev. D 51, 1362 (1995) arXiv:hep-ph/9408384]; for an extensive review on gauge mediation, see G. F. Giudice and R. Rattazzi, "Theories with gauge-mediated supersymmetry breaking," Phys. Rept. 322, 419 (1999) arXiv:hep-ph/9801271].

[29] L. Randall and R. Sundrum, "Out of this world supersymmetry breaking," Nucl. Phys. B 557, 79 (1999) arXiv:hep-th/9810155]; G. F. Giudice, M. A. Luty, H. Murayama and R. Rattazzi, "Gaugino mass without singlets," JHEP 9812, 027 (1998) arXiv:hep-ph/9810442; J. P. Conlon, M. Goodsell and E. Palti, "Anomaly mediation in superstring theory," arXiv:1008.4361 [hep-th].

[30] J. A. Bagger, T. Moroi and E. Poppitz, "Anomaly mediation in supergravity theories," JHEP 0004, 009 (2000) arXiv:hep-th/9911029]; K. Choi and H. P. Nilles, "The gaugino code," JHEP 0704, 006 (2007) arXiv:hep-ph/0702146

[31] K. Choi, C. S. Shin and H. P. Nilles, in preparation. 